\title{
Quantitative Analysis of Pathway Enrichment within Faba Bean Seeds RNA-Seq (Vicia faba L)
}

\author{
Shi Y. Yang1* Nuredin Habili², Qi Wu², Yunjie Wang ${ }^{3}$, Jiang Li ${ }^{4}$, Jeffrey G. Paull ${ }^{1}$ \\ ${ }^{1}$ School of Agriculture Food and Wine, The University of Adelaide, Waite Campus, Waite Road, Urrbrae, SA, Australia \\ ${ }^{2}$ The Australian Wine Research Institute, Urrbrae (Adelaide) SA, Australia \\ ${ }^{3}$ BGI-Shenzhen, BGI Tech Solutions Co., Ltd., Beishan Industrial Zone, Shenzhen, China \\ ${ }^{4}$ BIOZERON Biotech (Shenzhen) Co., Ltd., Shengzhen, China \\ Email: *shi.yang@adelaide.edu.au
}

How to cite this paper: Yang, S.Y., Habili, N., Wu, Q., Wang, Y.J., Li, J. and Paull, J.G. (2019) Quantitative Analysis of Pathway Enrichment within Faba Bean Seeds RNA-Seq ( Vicia faba L). American Journal of Plant Sciences, 10, 2305-2334. https://doi.org/10.4236/ajps.2019.1012161

Received: November 5, 2019

Accepted: December 28, 2019

Published: December 31, 2019

Copyright (c) 2019 by author(s) and Scientific Research Publishing Inc. This work is licensed under the Creative Commons Attribution International License (CC BY 4.0).

http://creativecommons.org/licenses/by/4.0/ Open Access

\begin{abstract}
Faba bean (Vicia faba L) seeds are an important source of plant protein for humans and animals. A total of 15,697 Differentially Expressed Genes (DEGs) with pathway annotation were discovered in RNA-Seq of the faba bean seeds. A total of 75 significant KEGG pathways abundance were discovered and 9 pathways were conserved within all genotypes. 41 significant pathways were found to be partially conserved within comparisons of 2 to 6 pairs of genotypes and 25 significant pathways were unique to single pairs of genotypes. There were 8 specific significant pathways discovered related to the faba bean seed Hydration Capacity trait and 9 specific significant pathways discovered related to the PSbMV seeds staining trait. The DEGs demonstrated the genetic distance between these varieties was confirmed by the breeding pedigree selection information and a PCA graph clearly illustrated the genetic distance within these genotypes.
\end{abstract}

\section{Keywords}

RNA-Seq (Quantification), Faba Bean (Vicia faba), Seed, Pathway Enrichment, Quantitative Analysis

\section{Introduction}

Faba bean (Vicia faba L) is one of the most important food and feed legume crops which provide a source of high protein for humans and animals, and it contributes to increased soil fertility through biological nitrogen fixation. It is an ancient crop and is cultivated by small holder Bronze age farms in the Mediterranean region [1]. Currently it is widely grown and the annual world production 
of faba bean was 4.8 million tonnes in 2017 (FAOSTAT 2018) [2]. It is a diploid species $(2 \mathrm{n}=12)$, with an exceptionally large genome of about $13.4 \mathrm{~Gb}$ [3], the largest genome in the grain legume family. RNA-Seq technology has been used to generate genome-wide transcriptome profiles across a wide range of plants including rice [4], maize [5] [6], chickpea [7] [8], field pea [9] [10], Raphanus sativus [11], faba bean [12] [13] [14] [15] [16] and pigeonpea [17]. These studies have described genome-wide gene expression levels in root, leaf, stem, T-cell and seed profiles. There have been reports of extensive pathway analyses in different plant species associated with different traits for six model plants: Arabidopsis thaliana, Oryza sativa, Zea mays, Solanum lycopersicum, Glycine max and Medicago. Expath database pathways of three plants: Arabidopsis thaliana, Oryza sativa, and Zea mays [18], AraPath database as molecular pathways of Arabidopsis [19], A. thaliana nutrient acquisition and general stress response pathways [20], modular expression pattern and new signaling pathways from motifs of Arabidopsis [21], responding to silver ions, cold, salt, drought and heat of Arabidopsis [22]; methylerythritol phosphate pathway associated genes with ceh1 mutuant of Arabidopsis [23], cadmium stress signaling controls in drought stress of rice [24], abiotic stress pathways and proteome analyses of maize [25], roots and leaves pathways associated with salt stress of cotton [26], phytohormone biosynthesis and signal transduction pathways of hazelnut ovules [27], pathway of secondary metabolism, transcription factors and transporters in response to methyl jasmonate of Lycoris aurea [28]. However, there is no quantitative pathways analysis reported for faba bean seeds or RNA-seq with associated related seed traits. Hence, we applied RNA-Seq (Quantification) technology to further study Differentially Expressed Genes (DEGs) to discover the KEGG pathway enrichment information within faba bean seeds, both pathways within all seeds in common and the pathways enrichment related to the typical seed traits of Hydration Capacity and Pea seed-borne mosaic virus (PSbMV).

\section{Materials and Methods}

\subsection{Materials}

The seeds of five faba bean varieties (Farah, Nura, PBA Rana, PBA Warda, and PBA Zahra) and one breeding line (AF06125) were used for the RNA-Seq (Quantification) technology analysis, with three biological samples per genotype. Seed was obtained from a field trial and was stored at room temperature until RNA was extracted 4 months after harvest. These varieties represent the diversity among Australian faba bean varieties and include diverse germplasm within their pedigrees [29].

The pedigrees and a brief description of the major traits of these varieties are bellowed:

Farah, Ascochyta blight resistant selection from Fiesta, medium size seed, early-mid flowering. Nura, selected from Icarus/Ascot, resistant to Ascochyta blight, small-medium size seed, mid flowering. 
Rana, selected from Manafest//611/Manafest, medium-large seed, mid flowering, low level of seed staining due to PSbMV.

PBA Warda, selected from IX038/IX004, resistant to rust, medium size seed, early flowering.

PBA Zahra, selected from 920/Farah, moderately resistant to Ascochyta blight, medium size seed.

AF06125, selected from 286/970//S95005/3/Icarus/Ascot//Farah, resistant to Ascochyta blight, low level of seed staining due to PSbMV, small-medium size seed, mid-season flowering, and high hydration capacity.

\subsection{Methods}

\section{RNA-Seq (Quantification) and Bioinformatics analysis}

RNA-Seq (Quantification) is used to analyze gene expression of certain biological objects under specific conditions [30] [31].

\subsubsection{Total RNA Extraction}

RNA extraction using RNeasy kit (Qiagen, Australia) according to the manufacturer's instructions. The RNA-Seq experimental process shows the steps for the experimental pipeline. During the QC step, Agilent 2100 Bioanaylzer was used to qualify and quantify the sample library. The library products were ready for sequencing on the Ion Proton platform performed by the Beijing Genomics Institute (BGI-Shenzhen).

\subsubsection{RNA Solutions Preparation and Sequencing}

The total RNA samples were first treated with DNase I to degrade any possible DNA contamination. Then the mRNA was enriched by using the oligo (dT) magnetic beads. Mixed with the fragmentation buffer, the mRNA was fragmented into short fragments (about $200 \mathrm{bp}$ ). Then the first strand of cDNA was synthesized by using random hexamer-primed reverse transcription. Buffer, dNTPs, RNase $\mathrm{H}$ and DNA polymerase I were added to synthesize the second strand. The double strand cDNA was purified with magnetic beads. End reparation was then performed. After the previous step, adaptors were ligated to the end of these fragments. Next, ligation products were selected by size and purified on TAE-agarose gel. Finally, the fragments were enriched by PCR amplification, then purified by magnetic beads and dissolved in the appropriate amount of Epstein-Barr solution. During the QC step, Agilent 2100 Bioanaylzer was used to qualify and quantify the sample library. The library products were then ready for sequencing via Ion Proton platform.

\subsection{Bioinformatics Analysis Pipeline}

Primary sequencing data that produced by Ion Proton, called as raw reads, were subjected to quality control that determined if a resequencing step was needed. After quality control, raw reads were filtered into clean reads which were transformed to fq format, and aligned to the reference sequences at the same time. 
QC of alignment was performed to determine if resequencing was needed. The alignment data was utilized to calculate distribution of reads on reference genes and mapping ratio. If alignment result passed QC, we proceeded with downstream analysis including gene expression and deep analysis based on gene expression such as PCA/correlation/screening differentially expressed genes, and further perform deep analysis based on DEGs, including cluster analysis, Gene Ontology analysis and Pathway enrichment analysis.

\subsubsection{Sequencing Data Assess}

The original image data was transferred into sequence data via base calling, which is defined as raw data or raw reads and saved as BAM file. As the raw reads may contain low quality reads or adaptor sequences, preprocessing was necessary before starting further analysis.

\subsubsection{Raw Data Statistics}

As there are some adaptor sequences and/or low quality reads present in the raw reads data filtering was carried out to obtain high quality reads as the clean reads (clean data). Filtering steps were as follows:

1) Remove reads where length was less than threshold;

2) Trim reads adapter, if length of trimmed reads was less than threshold, then remove it;

3) Calculate the average quality of 15 bases from 3 ' end until the average quality was larger than 10 , then trim the bases that have been counted.

After filtering, the remaining reads are called "clean reads" and used for downstream bioinformatics analysis.

\subsubsection{Base Composition, Quality and Length of Clean Data}

We performed quality control on clean data through drawing base composition, quality and length distribution charts. ATGC base content refers to the proportion of the total bases of the four types of bases. Through detecting the content of ATGC each base to measure the stability of library and sequencing eligibility. The nucleotide distributions at each position were stable under the normal circumstances, non-AT or GC separation. Bases quality reflects the accuracy which can be affected by RNA-Sequencing, reagents or sample quality. If the percentage of the bases with low quality is low, then it indicated the sequencing quality is good.

\subsubsection{Alignment Assessment}

After data quality statistics, clean reads were mapped to gene reference and/or genome reference set.

\subsubsection{Alignment Statistics}

We used TMAP to map clean reads to gene reference and/or genome reference. In general, the higher ratio of alignment, indicated the closer the genetic relationship between the samples and the reference faba bean species. 


\subsubsection{Sequencing Saturation Analysis}

Sequence saturation analysis was used to measure the sequencing data of the sample. The number of detected genes increased with an increase in the number of reads. However, when the number of reads reaches a certain amount, the growth curve of detected genes flattens, which indicates that the number of detected genes tends to saturation.

\subsubsection{Reads Distribution on Gene}

If the randomness is good, the reads in every position would be evenly distributed. If the randomness is poor, reads preference to specific gene region will directly affect subsequent bioinformatics analysis. We used the distribution of reads on the reference genes to evaluate the randomness. A distribution of reads position on genes shows that the randomness of RNA fragmentation is better than cDNA fragmentation [32]. Therefore, we used the mRNA fragments to construct the library in the experiment.

\subsubsection{Visualization of Genome Alignment}

We provide genome alignment data in BAM format and recommend using IGV (Integrative Genomics Viewer) tool to visualize BAM file in different scales. IGV supports loading of multiple samples to do comparison in the same scale, and can view distribution of reads on the Exon, Intron, UTR, and Intragenic regions, which makes it very convenient and intuitional.

\subsubsection{Gene Expression}

Expression levels of individual Unigenes were quantified using the software package Sailfish [33]. Sailfish quantifies genes expression by k-mer, first, built index files use reference and length of $\mathrm{k}$-mer, then computes maximum likelihood abundance estimates using the Expectation-Maximization (EM) algorithm for its statistical model to determine which transcripts are isoforms of the same gene. Expression level was measured in reads per kilobase per million mapped reads (RPKM) according to Equation (1):

$$
\mathrm{RPKM}=\frac{10^{6} \mathrm{C}}{N L / 10^{3}}
$$

where $C$ is the number of reads that are uniquely aligned to a specified gene (A), $N$ is the total number of reads uniquely aligned to all genes and $L$ is the length of the specified gene (A) in bases. The RPKM values can be directly used for comparing the difference of gene expression among samples. If there was more than one transcript for a gene, the longest one was used to calculate its expression level and coverage. Linear correlation of RPKM values was used to assess the robustness of experimental comparisons made between genotypes.

\subsubsection{Deep Analysis of Gene Expression}

For multiple samples, we can do more deep analysis based on gene expression to do a comprehensive assess on the whole project. 


\subsubsection{Correlation between Genotypes}

We calculated the correlation value between each two genotypes based on RPKM results. According to the standard that Encode plan recommends, the square of the correlation value should be $\geq 0.92$ (under ideal experiment environment and with reasonable samples).

\subsubsection{Cluster Tree of All Samples}

The distances of expressed genes in samples were calculated by Euclidean method. Meanwhile, the algorithm of Sum of Squares of Deviations was used to calculate the distance between samples so that a cluster tree could be built.

\subsubsection{Cluster Analysis of Gene Expression}

Genes with similar expression patterns usually have same the functional correlation. We performed cluster analysis of gene expression patterns with cluster [34] [35] and java Treeview software [36].

\subsubsection{PCA Analysis}

Principal component analysis (PCA) can reduce the complexity of the data, and dig deep into the relation between sample size and variation. The basic principle is that diverse samples have different measurements; PCA identifies the main factors of observed value differences, considering all the factors are combined and sorted according to importance. In PCA graph it can see the distance of the relationship between each sample, including visual effect of clusters groups. The PCA analysis is presented as a figure where each dot represents the genotype on the principal component value.

\subsubsection{Screening of Differentially Expressed Genes (DEGs)}

Screening for DEGs was based on the Poisson distribution method described by Audic \& Claverie [37] and corrected P-values using the Bonferroni method [38]. Since DEGs analysis generates a large multiplicity of problems in which thousands of hypotheses (is gene $\mathrm{x}$ differentially expressed between the two groups) are tested simultaneously, correction for false positive (type I errors) and false negative (type II) errors was performed using the False Discovery Rate (FDR) method [39]. We used FDR $\leq 0.001$ and the absolute value of $\log _{2}$ Ratio $\geq 1$ as the threshold to judge the significance of gene expression difference.

DEGs screening is aimed to find differentially expressed genes between (within) samples and perform further function analysis on them. We provide three optional methods for screening analysis of differentially expressed genes, respectively: Based on the analysis method of the Poisson distribution; Based on Noiseq package method; Based on EBSeq package method.

According to the analysis method of the Poisson distribution which screening DEGs between two genotypes. Referring to the significance of digital gene expression profiles, we have developed a strict algorithm to identify differentially expressed genes between two samples. Denote the number of unambiguous clean tags (which means reads in RNA-Seq) from gene A as $\mathrm{x}$, given every gene's 
expression occupies only a small part of the library, $\mathrm{x}$ yields to the Poisson distribution with Equation (2):

$$
p(x)=\frac{\mathrm{e}^{-\lambda} \lambda^{x}}{x !}(\lambda \text { is the real transcripts of the gene })
$$

The total clean tag number of sample 1 is $N_{1}$, and total clean tag number of sample 2 is $N_{2}$; gene A holds x tags in sample 1 and y tags in sample 2. The probability of gene A being expressed equally between two samples can be calculated with Equation (3):

$$
\begin{gathered}
2 \sum_{i=0}^{i=y} p(i \mid x) \\
\text { or } 2 \times\left(1-\sum_{i=0}^{i=y} p(i \mid x)\right)\left(\text { if } \sum_{i=0}^{i=y} p(i \mid x)>0.5\right) \\
p(y \mid x)=\left(\frac{N_{2}}{N_{1}}\right)^{y} \frac{(x+y) !}{x ! y !\left(1+\frac{N_{2}}{N_{1}}\right)^{(x+y+1)}}
\end{gathered}
$$

We do correction on P-value corresponds to differential gene expression test using Bonferroni method. Since DEG analysis generates a large multiplicity problems in which thousands of hypotheses (is gene $\mathrm{x}$ differentially expressed between the two groups) are tested simultaneously, correction for false positive (type I errors) and false negative (type II) errors are performed using FDR method. Assume that we have picked out $\mathrm{R}$ differentially expressed genes in which $\mathrm{S}$ genes really show differential expression and the other $\mathrm{V}$ genes are false positive. If we decide that the error ratio " $Q=V / R$ " must stay below a cutoff (e.g. 5\%), we should preset the FDR to a number no larger than 0.05 . We use "FDR $\leq 0.001[40]$ and the absolute value of $\log _{2}$ Ratio $\geq 1$ " as the threshold to judge the significance of gene expression difference. More stringent criteria with smaller FDR and bigger fold-change value can be used to identify DEGs.

Based on Noiseq package method (screening DEGs among two groups which contain biological replicates) Noiseq method [41] can screen differentially expressed genes between two groups, showing a good performance when comparing it to other differential expression methods, like Fisher's Exact Test (FET), edgeR, DESeq and baySeq. Noiseq maintains good True Positive and False Positive rates when increasing sequencing depth, while most other methods show poor performance. In addition, Noiseq models the noise distribution from the actual data, so it can better adapt to the size of the data set, and is more effective in controlling the rate of false discoveries.

First, Noiseq uses the sample's gene expression in each group to calculate $\log _{2}$ (foldchange) $M$ and absolute different value $D$ of all pair conditions to build noise distribution model with Equation (4):

$$
M^{i}=\log _{2}\left(\frac{x_{1}^{i}}{x_{i}^{i}}\right) \text { and } D^{i}=\left|x_{1}^{i}-x_{12}^{i}\right|
$$


Second, for gene A, Noiseq computes its average expression "Control-avg" in control group and average expression "Treat-avg" in treatment group. Then the foldchange $\left(M_{A}=\log _{2}((\right.$ Controlavg $) /($ Treat-avg $\left.))\right)$ and absolute different value $D\left(D_{A}=\mid\right.$ Control-avg-Treat-avg $\left.\mid\right)$ will be derived. If $M_{A}$ and $D_{A}$ diverge from noise distribution model markedly, gene A will be defined as a DEG. There is a probability value to assess how $M_{A}$ and $D_{A}$ both diverge from noise distribution model with Equation (5):

$$
P_{A}=P\left(M_{A} \geq\{M\} \& \& D_{A} \geq\{D\}\right)
$$

Finally, we screen differentially expressed genes according to the following criteria: Fold change $\geq 2$ and diverge probability $\geq 0.8$.

\subsubsection{Deep Analysis of DEGs}

Cluster analyses of DEGs with similar expression patterns usually have the same functional correlation. We perform cluster analysis of DEGs with cluster and java Treeview software [36]. Expression differences are shown in different colors. Red means up regulation and green means down regulation.

\subsubsection{Pathway Enrichment Analysis of DEGs}

Genes usually interact with each other to play roles in certain biological functions. Pathway-based analysis helps to further understand genes biological functions. KEGG (Kyoto Encyclopedia of Genes and Genomes) [38] pathways, the major public pathway-related database, is used to perform pathway enrichment analysis of DEGs. This analysis identifies significantly enriched metabolic pathways or signal transduction pathways in DEGs comparing with the whole genome background. The calculating formula as where $\mathrm{N}$ is the number of all genes with KEGG annotation, $\mathrm{n}$ is the number of DEGs in $\mathrm{N}$, M is the number of all genes annotated to specific pathways, and $\mathrm{m}$ is the number of DEGs in $\mathrm{M}$. The calculated $p$-value goes through Bonferroni Correction [40] method, taking corrected $p$-value $<0.05$ as a threshold. Discovered the most significant pathways of faba bean seed RNA-Seq, allows us to see their detailed pathway information in the KEGG database. The pathways showed with their up-regulated genes which are marked with red borders and down-regulated genes with green borders, Non-change genes are marked with black borders. A scatter plot for KEGG enrichment results was generated to display the top 20 enriched pathways (See Figure 1 and Supplementary Figures S1-S14). Rich Factor is the ratio of DEGs numbers annotated in this pathway to all gene numbers. Greater Rich Factor means greater intensiveness. Q-value is corrected $p$-value ranging from $0 \sim 1$, and a lower value means greater intensiveness.

\subsection{Validation of Hydration Capacity and PSbMV Index}

1) Hydration Capacity testing was undertaken on these 6 genotypes from samples obtained from field trials conducted at Charlick Experimental farm, Strathalbyn and at Turretfield or Freeling, South Australia, from 2007 to 2017, 


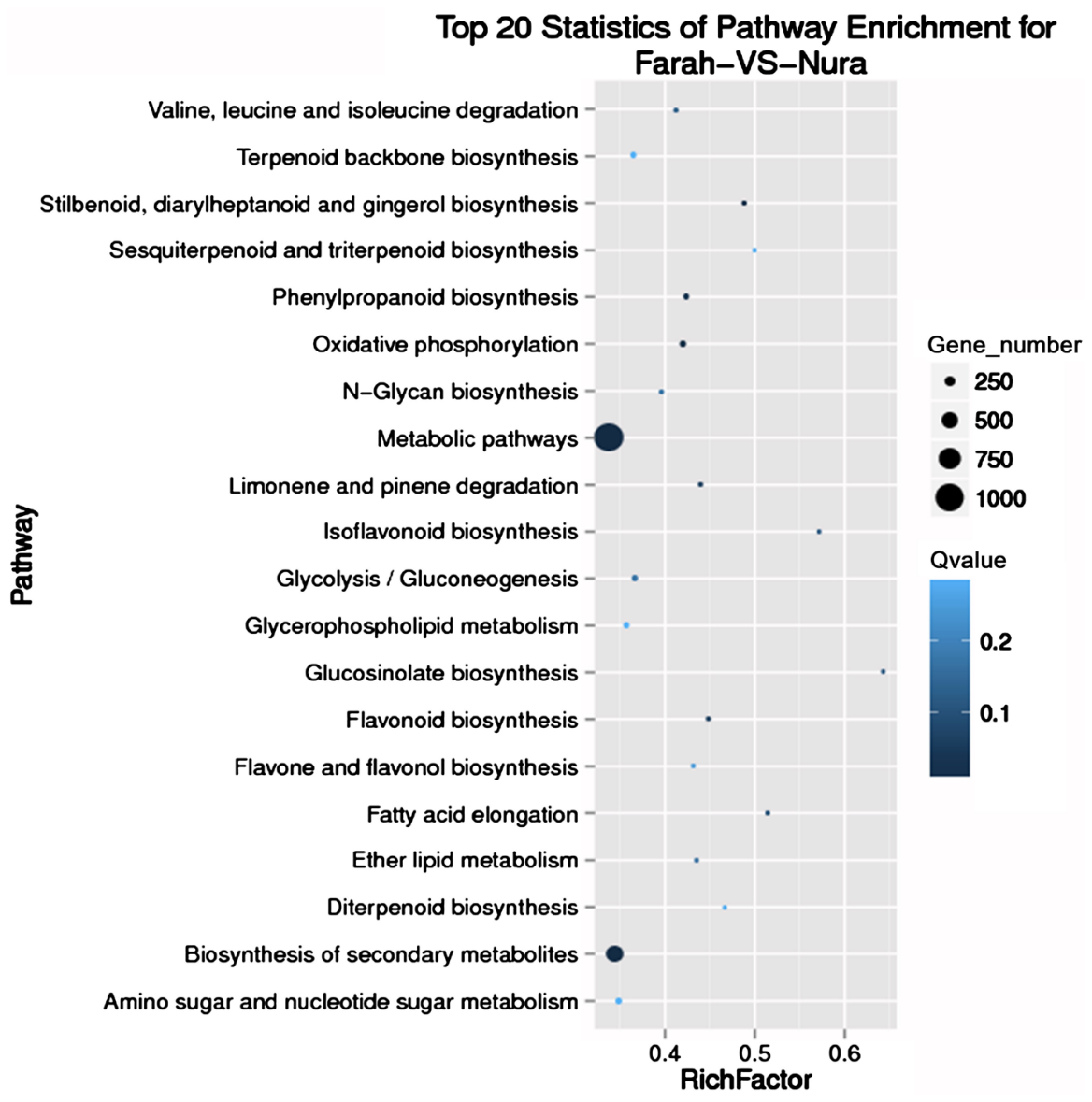

Figure 1. The top 20 pathways enriched in faba bean seed, including gene number and Q value, for the comparison of Farah versus Nura.

with three replicated per genotype/experiment. The standard Hydration Capacity test consists of soaking 50 seeds in $150 \mathrm{ml} \mathrm{RO}$ water at $22^{\circ} \mathrm{C}$ for 16 hours and determining the $\%$ change in weight due to uptake of water.

2) 20 samples of each faba bean genotype were sown in a growth room in 2018 and inoculated with PSbMV virus (mechanical inoculation with macerated infected field pea leaves mixed with carborundum powder and rubbed on the faba bean leaves) when they were at the two leaf growth stage. Faba bean leaves were collected 6 weeks after inoculation to validate infection by PSbMV using both ELISA assay and RT-PCR sequence. Seeds were harvested at maturity and assessed for staining due to PSbMV. Seeds were assigned to one of 6 categories rated as 0 for no staining to 5 for very severe staining and an index $F X$ of overall staining for the individual sample was calculated by the following Equation (6):

$$
F X=\frac{n_{0} * 0+n_{1} * 1+n_{2} * 2+n_{3} * 3+n_{4} * 4+n_{5} * 5}{5 * N} * 100
$$

where $n=$ the number of seeds within a category, $N=$ the total number of seeds.

\section{Results and Discussion}

Genes interact with each other to play roles in certain biological functions. 
Pathway-based analysis helps to further understand the genes biological functions. KEGG (Kyoto Encyclopedia of Genes and Genomes) is the major public pathway-related database (http://www.kegg.jp/kegg/kegg1.html), which is used to perform pathway enrichment analysis of DEGs. This analysis identifies the most significant enriched pathway in DEGs as compared with the whole genome. Detecting the most significantly enriched pathway of DEGs, allowed us to see detailed pathway information in the KEGG database, no matter if the genes are up-regulated or down-regulated. We generated a scatter plot for KEGG enrichment results. Rich Factor is the ratio of number of differentially expressed genes in this pathway to the number of all genes annotated in there. The greater the Rich Factor means the greater the degree of pathway enrichment. The $\mathrm{Q}$ value is the corrected $p$ value with the range $0-1$, where a lower value means greater intensiveness. The top 20 pathways enriched in comparisons between individual genotypes and between phenotype groupings are presented in each comparison (See Figure 1 for Farah vs Nura and Supplementary files Figures S1-S14). These results indicate that most significant enriched pathway is Metabolic pathways (ko01100) followed by the Biosynthesis of secondary metabolites (ko01110); Plant hormone signal transduction (ko04075); Plant - pathogen interaction (ko04626); $\mathrm{ABC}$ transporters (ko02010); Zeatin biosynthesis (ko00908); Stilbenoid, diarylheptanoid and gingerol biosynthesis (ko00945); Phenylpropanoid biosynthesis (ko00940); Phenylalanine, tyrosine and tryptophan biosynthesis (ko00400); Pentose and glucuronate interconversions (ko00040); N-Glycan biosynthesis (ko00510); mismatch repair (ko03430); Limonene and pinene degradation (ko00903); Isoflavonoid biosynthesis (ko00943); Glycine, serine and threonine metabolism (ko00260); Glycerolipid metabolism (ko00561); Flavonoid biosynthesis (ko00941); Fatty acid biosynthesis (ko00061); Caffeine metabolism (ko00232) and Base excision repair (ko03410).

\subsection{Common KEGG Pathways Abundance Discovered within Faba Bean Seeds RNA-Seq}

A total of 47,621 expressed genes were identified in faba bean seeds RNA-Seq [16]. There were 15,697 significant Differentially Expressed Genes (DEGs) with pathway annotation. A total of 266 significant or highly significant enriched pathways were discovered in faba bean seeds by single variety pair comparisons. This was reduced to 75 unique abundance pathways after removal of redundancies (See Table 1 and Table 2).

In the comparison of individual pairs of genotypes, the most common pathways were Metabolic pathways (ko01100) and Biosynthesis of secondary metabolites (ko01110) where commonality of the pathways was significant to highly significant for 14 out of a total of 15 (93.3\%) of all pairwise comparisons. Only one pair comparison (PBAZahra versus AF06125) was not significant within these two pathways. The second most common pathway was Zeatin biosynthesis (ko00908) which was significant for 13 out of 15 (86.7\%) pair comparisons. Four pathways were significant for 9 out of $15(60 \%)$ pair comparisons: 
Table 1. KEGG Pathway DEGs numbers within faba bean seeds RNA-Seq.

\begin{tabular}{|c|c|c|c|c|c|c|}
\hline \multirow{2}{*}{$\begin{array}{l}\text { Pathway } \\
\text { No }\end{array}$} & \multirow[t]{2}{*}{ KEGG Pathway } & \multicolumn{2}{|c|}{$\begin{array}{l}\text { All DEGs with pathway } \\
\text { annotation (15697) }\end{array}$} & \multirow{2}{*}{$\begin{array}{l}\text { Pathway } \\
\text { ID }\end{array}$} & \multirow{2}{*}{$\begin{array}{l}\text { No of pairs } \\
\text { in common }\end{array}$} & \multirow[t]{2}{*}{ Significance } \\
\hline & & DEGs No & Percentage (\%) & & & \\
\hline 1 & Metabolic pathways & 3127 & 19.92 & ko01100 & 14 & ${ }^{*}(1),{ }^{* *}(7),{ }^{* * *}(6)$ \\
\hline 2 & Biosynthesis of secondary metabolites & 1592 & 10.14 & ko01110 & 14 & ${ }^{*}(2),{ }^{* *}(6),{ }^{* * *}(6)$ \\
\hline 3 & Zeatin biosynthesis & 121 & 0.77 & ko00908 & 13 & ${ }^{*}(5),{ }^{* *}(4),{ }^{* * *}(4)$ \\
\hline 4 & alpha-Linolenic acid metabolism & 79 & 0.50 & ko00592 & 9 & ${ }^{*}(5),{ }^{* *}(2),{ }^{* * *}(2)$ \\
\hline 5 & Limonene and pinene degradation & 91 & 0.58 & ko00903 & 9 & ${ }^{*}(5), * *(3), * * *(1)$ \\
\hline 6 & Phenylpropanoid biosynthesis & 144 & 0.92 & ko00940 & 9 & ${ }^{\star}(2),{ }^{* *}(4),{ }^{* * *}(3)$ \\
\hline 7 & Terpenoid backbone biosynthesis & 148 & 0.94 & ko00900 & 9 & ${ }^{*}(4),{ }^{* *}(3),{ }^{* *}(2)$ \\
\hline 8 & Plant hormone signal transduction & 589 & 3.75 & ko04075 & 8 & ${ }^{*}(4),{ }^{* *}(3),{ }^{* *}(1)$ \\
\hline 9 & Stilbenoid, diarylheptanoid and gingerol biosynthesis & 86 & 0.55 & ko00945 & 8 & ${ }^{*}(3),{ }^{* *}(2),{ }^{* *}(3)$ \\
\hline 10 & Flavone and flavonol biosynthesis & 51 & 0.32 & ko00944 & 6 & ${ }^{*}(2),{ }^{* *}(2),{ }^{* *}(2)$ \\
\hline 11 & Phenylalanine metabolism & 74 & 0.47 & ko00360 & 6 & $*(5),{ }^{* * *}(1)$ \\
\hline 12 & Plant-pathogen interaction & 543 & 3.46 & ko04626 & 6 & ${ }^{*}(3),{ }^{* *}(1),{ }^{* *}(2)$ \\
\hline 13 & Caffeine metabolism & 7 & 0.04 & ko00232 & 5 & ${ }^{*}(3),{ }^{* *}(2)$ \\
\hline 14 & Flavonoid biosynthesis & 87 & 0.55 & ko00941 & 5 & ${ }^{*}(4),{ }^{* *}(1)$ \\
\hline 15 & Fructose and mannose metabolism & 107 & 0.68 & ko00051 & 5 & * \\
\hline 16 & Linoleic acid metabolism & 36 & 0.23 & ko00591 & 5 & ${ }^{\star}(4),{ }^{* * *}(1)$ \\
\hline 17 & Mismatch repair & 128 & 0.82 & ko03430 & 5 & * \\
\hline 18 & N-Glycan biosynthesis & 106 & 0.68 & ko00510 & 5 & ${ }^{*}(3),{ }^{* *}(2)$ \\
\hline 19 & Pentose and glucuronate interconversions & 92 & 0.59 & ko00040 & 5 & ${ }^{*}(2),{ }^{* *}(3)$ \\
\hline 20 & Sesquiterpenoid and triterpenoid biosynthesis & 22 & 0.14 & ko00909 & 5 & ${ }^{*}(4),{ }^{* *}(1)$ \\
\hline 21 & $\mathrm{ABC}$ transporters & 152 & 0.97 & ko02010 & 4 & ${ }^{*}(2),{ }^{* *}(1), * *(1)$ \\
\hline 22 & Diterpenoid biosynthesis & 30 & 0.19 & ko00904 & 4 & * \\
\hline 23 & Ether lipid metabolism & 62 & 0.39 & ko00565 & 4 & * \\
\hline 24 & Fatty acid biosynthesis & 50 & 0.32 & ko00061 & 4 & ${ }^{\star}(2),{ }^{* *}(2)$ \\
\hline 25 & Fatty acid elongation & 35 & 0.22 & ko00062 & 4 & ${ }^{*}(3),{ }^{* *}(1)$ \\
\hline 26 & Galactose metabolism & 134 & 0.85 & ko00052 & 4 & * \\
\hline 27 & Glycosphingolipid biosynthesis - ganglio series & 31 & 0.20 & ko00604 & 4 & $*(2),{ }^{* *}(2)$ \\
\hline 28 & Photosynthesis - antenna proteins & 16 & 0.10 & ko00196 & 4 & * \\
\hline 29 & Base excision repair & 113 & 0.72 & ko03410 & 3 & * \\
\hline 30 & Biosynthesis of unsaturated fatty acids & 45 & 0.29 & ko01040 & 3 & ${ }^{*}(2),{ }^{* *}(1)$ \\
\hline 31 & DNA replication & 130 & 0.83 & ko03030 & 3 & ${ }^{\star}(1),{ }^{* *}(2)$ \\
\hline 32 & Fatty acid metabolism & 106 & 0.68 & ko00071 & 3 & * \\
\hline 33 & Glucosinolate biosynthesis & 14 & 0.09 & ko00966 & 3 & ${ }^{\star}(1),{ }^{* *}(2)$ \\
\hline 34 & Glutathione metabolism & 109 & 0.69 & ko00480 & 3 & ${ }^{*}(2),{ }^{* *}(1)$ \\
\hline 35 & Glycerolipid metabolism & 126 & 0.80 & ko00561 & 3 & * \\
\hline 36 & Glycerophospholipid metabolism & 182 & 1.16 & ko00564 & 3 & * \\
\hline 37 & Glycine, serine and threonine metabolism & 104 & 0.66 & ko00260 & 3 & ${ }^{\star}(2),{ }^{\star *}(1)$ \\
\hline
\end{tabular}




\section{Continued}

\begin{tabular}{|c|c|c|c|c|c|c|}
\hline 38 & Isoflavonoid biosynthesis & 21 & 0.13 & ko00943 & 3 & ${ }^{\star}(1),{ }^{\star \star}(2)$ \\
\hline 39 & Oxidative phosphorylation & 212 & 1.35 & ko00190 & 3 & $* *(1), * * *(2)$ \\
\hline 40 & Pentose phosphate pathway & 93 & 0.59 & ko00030 & 3 & ${ }^{\star}(2),{ }^{* *}(1)$ \\
\hline 41 & Starch and sucrose metabolism & 317 & 2.02 & ko00500 & 3 & ${ }^{*}(2),{ }^{* *}(1)$ \\
\hline 42 & Ubiquinone and other terpenoid-quinone biosynthesis & 61 & 0.39 & ko00130 & 3 & * \\
\hline 43 & Amino sugar and nucleotide sugar metabolism & 241 & 1.54 & ko00520 & 2 & * \\
\hline 44 & Benzoxazinoid biosynthesis & 16 & 0.10 & ko00402 & 2 & ${ }^{*}(1),{ }^{* *}(1)$ \\
\hline 45 & Cutin, suberine and wax biosynthesis & 29 & 0.18 & ko00073 & 2 & ${ }^{\star}(1),{ }^{\star \star}(1)$ \\
\hline 46 & Cyanoamino acid metabolism & 51 & 0.32 & ko00460 & 2 & * \\
\hline 47 & Cysteine and methionine metabolism & 97 & 0.62 & ko00270 & 2 & ${ }^{*}(1),{ }^{* *}(1)$ \\
\hline 48 & Riboflavin metabolism & 22 & 0.14 & ko00740 & 2 & ${ }^{*}(1),{ }^{* *}(1)$ \\
\hline 49 & RNA degradation & 423 & 2.69 & ko03018 & 2 & * \\
\hline 50 & Sphingolipid metabolism & 80 & 0.51 & ko00600 & 2 & * \\
\hline 51 & Phenylalanine, tyrosine and tryptophan biosynthesis & 69 & 0.44 & ko00400 & 1 & * \\
\hline 52 & Ascorbate and aldarate metabolism & 75 & 0.48 & ko00053 & 1 & * \\
\hline 53 & Indole alkaloid biosynthesis & 8 & 0.05 & ko00901 & 1 & * \\
\hline 54 & Porphyrin and chlorophyll metabolism & 83 & 0.53 & ko00860 & 1 & * \\
\hline 55 & Endocytosis & 256 & 1.63 & ko04144 & 1 & * \\
\hline 56 & Selenocompound metabolism & 38 & 0.24 & ko00450 & 1 & * \\
\hline 57 & Aminoacyl-tRNA biosynthesis & 158 & 1.01 & ko00970 & 1 & * \\
\hline 58 & Arachidonic acid metabolism & 23 & 0.15 & ko00590 & 1 & * \\
\hline 59 & Pantothenate and CoA biosynthesis & 55 & 0.35 & ko00770 & 1 & * \\
\hline 60 & RNA polymerase & 126 & 0.80 & ko03020 & 1 & * \\
\hline 61 & Folate biosynthesis & 37 & 0.24 & ko00790 & 1 & * \\
\hline 62 & Monoterpenoid biosynthesis & 10 & 0.06 & ko00902 & 1 & * \\
\hline 63 & Other glycan degradation & 55 & 0.35 & ko00511 & 1 & * \\
\hline 64 & Nucleotide excision repair & 200 & 1.27 & ko03420 & 1 & * \\
\hline 65 & Photosynthesis & 33 & 0.21 & ko00195 & 1 & * \\
\hline 66 & Purine metabolism & 350 & 2.23 & ko00230 & 1 & * \\
\hline 67 & Tropane, piperidine and pyridine alkaloid biosynthesis & 27 & 0.17 & ko00960 & 1 & ** \\
\hline 68 & Glycolysis/Gluconeogenesis & 210 & 1.34 & ko00010 & 1 & * \\
\hline 69 & Glyoxylate and dicarboxylate metabolism & 80 & 0.51 & ko00630 & 1 & * \\
\hline 70 & Synthesis and degradation of ketone bodies & 23 & 0.15 & ko00072 & 1 & * \\
\hline 71 & Valine, leucine and isoleucine degradation & 97 & 0.62 & ko00280 & 1 & $* *$ \\
\hline 72 & Alanine, aspartate and glutamate metabolism & 85 & 0.54 & ko00250 & 1 & * \\
\hline 73 & Circadian rhythm - plant & 124 & 0.79 & ko04712 & 1 & * \\
\hline 74 & Glycosphingolipid biosynthesis - globo series & 14 & 0.09 & ko00603 & 1 & $* *$ \\
\hline 75 & Pyruvate metabolism & 150 & 0.96 & ko00620 & 1 & * \\
\hline
\end{tabular}

Note: ${ }^{*}$ means $0.01 \leq P \leq 0.05,{ }^{* *}$ means $0.001 \leq P \leq 0.01,{ }^{* * *}$ means $P<0.001$. () If the significance level is different in the same KEGG pathway within different pairs, the number in the bracket represents the number of pairs at the same level of significance of faba bean seeds in common in total of 15 pairs analyzed. 
Table 2. Significant Pathways number in different pairs and related traits within faba bean seeds RNA-Seq.

\begin{tabular}{|c|c|c|c|c|}
\hline \multirow{2}{*}{ Pair comparison } & \multicolumn{3}{|c|}{ Number of Significant pathways } & \multirow{2}{*}{$\begin{array}{l}\text { Sub } \\
\text { Total }\end{array}$} \\
\hline & $* * * P<0.001$ & ${ }^{* *} 0.001 \leq P \leq 0.01$ & ${ }^{*} 0.01 \leq P \leq 0.05$ & \\
\hline 1. AF06125-VS-Farah & 2 & 8 & 9 & 19 \\
\hline 2. AF06125-VS-Nura & 1 & 9 & 7 & 17 \\
\hline 3. AF06125-VS-PBARana & 2 & 9 & 10 & 21 \\
\hline 4. AF06125-VS-PBAWarda & 3 & 5 & 9 & 17 \\
\hline 5. Farah-VS-Nura & 5 & 7 & 11 & 23 \\
\hline 6. Farah-VS-PBARana & 3 & 6 & 11 & 20 \\
\hline 7. Farah-VS-PBAWarda & 3 & 5 & 13 & 21 \\
\hline 8. Nura-VS-PBARana & 3 & 8 & 11 & 22 \\
\hline 9. Nura-VS-PBAWarda & 2 & 6 & 17 & 25 \\
\hline 10. PBARana-VS-PBAWarda & 3 & 5 & 10 & 18 \\
\hline 11. PBAZahra-VS-AF06125 & 1 & 1 & 4 & 6 \\
\hline 12. PBAZahra-VS-Farah & 2 & 4 & 6 & 12 \\
\hline 13. PBAZahra-VS-Nura & 3 & 4 & 7 & 14 \\
\hline 14. PBAZahra-VS-PBARana & 3 & 4 & 8 & 15 \\
\hline 15. PBAZahra-VS-PBAWarda & 4 & 5 & 7 & 16 \\
\hline Total & 40 & 86 & 140 & 266 \\
\hline \multicolumn{5}{|l|}{ Trait comparison } \\
\hline Lowest Hydration Capacity-VS & 1 & 4 & 4 & 9 \\
\hline \multicolumn{5}{|l|}{ Highest Hydration Capacity } \\
\hline $\begin{array}{l}\text { Intermediate Hydration } \\
\text { Capacity-VS- }\end{array}$ & & 1 & 2 & 3 \\
\hline \multicolumn{5}{|l|}{ Highest Hydration Capacity } \\
\hline PSbMV Low seed staining-VS- & 1 & 3 & 7 & 11 \\
\hline High seed staining & & & & \\
\hline
\end{tabular}

Note: ${ }^{*}$ means $0.01 \leq P \leq 0.05,{ }^{* *}$ means $0.001 \leq P \leq 0.01,{ }^{* * *}$ means $P<0.001$.

alpha-Linolenic acid metabolism (ko00592), Limonene and pinene degradation (ko00903), Phenylpropanoid biosynthesis (ko00940) and Terpenoid backbone biosynthesis (ko00900). Plant hormone signal transduction (ko04075) and Stilbenoid, diarylheptanoid and gingerol biosynthesis (ko00945) were significant for 8 out of 15 (53.3\%) pairs comparison. Flavone and flavonol biosynthesis (ko00944); Phenylalanine metabolism (ko00360) and Plant-pathogen interaction (ko04626) were significant for the comparison of 6 out of 15 (40\%) pairs.

Many pathways displayed a lower level of commonality among pairs of genotypes (Table 1), with eight pathways being significant for 5 out of 15 (33.3\%) comparisons, and an additional 8 pathways significant for 4 (26.7\%) compari- 
son. Fourteen pathways were significant for 3 (20\%), and eight pathways significant for 2 (13.3\%) of comparisons. There were 25 pathways that were significant for only a single pair of genotypes.

\subsection{Trait Related KEGG Specific Pathways Abundance Discovered within Faba Bean Seed}

The comparison of genotypes grouped on the basis of particular seed traits was much more specific than the comparison of individual genotype pairs. Regarding the seed trait Hydration Capacity the comparison between the Lowest and the Highest groups identified 5 pathways where abundance was highly significant: Zeatin biosynthesis (ko00908), Ribosome biogenesis in eukaryotes (ko03008), RNA degradation (ko03018), Terpenoid backbone biosynthesis (ko00900), and Homologous recombination (ko003440), while three pathways were significant: Biosynthesis of secondary metabolites (ko01110), Porphyrin and chlorophyll metabolism (ko00860), and Stilbenoid, diarylheptanoid and gingerol biosynthesis (ko00945) (See Figure 1). For the comparison between Intermediate Hydration Capacity and Highest Hydration Capacity, there was only one pathway that was highly significant: Base excision (ko03410), and there were two significant pathways: Cutin, suberine and wax biosynthesis (ko00073) and Zeatin biosynthesis (ko00908) (See Figure 2(a) and Figure 2(b)).

Regarding the trait of Pea seed-borne mosaic virus ( PSbMV) and comparison of Low seed staining versus High seed staining genotypes, there were four highly significant pathways: Zeatin biosynthesis (ko00908), Valine, leucine and isoleucine degradation (ko00280), Base excision repair (ko03410) and Phenylpropanoid biosynthesis (ko00940) and seven significant pathways: Biosynthesis of secondary

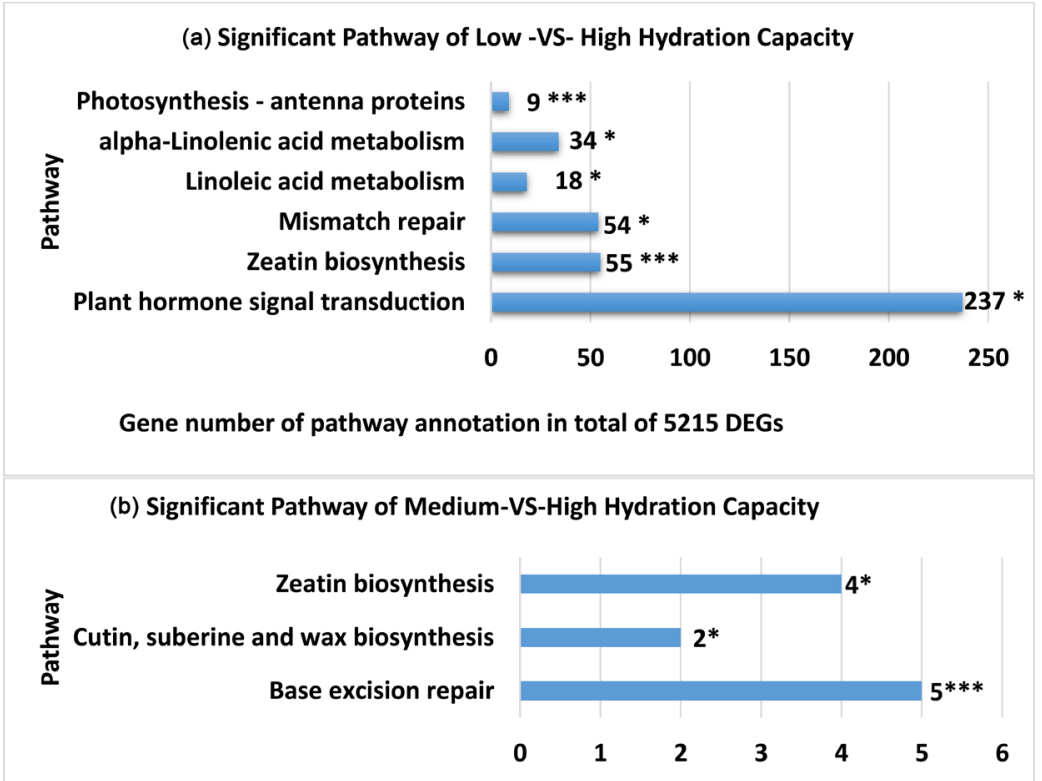

Figure 2. Significant pathways in faba bean seeds for comparisons of (a) varieties with Low versus High Hydration Capacity and (b) varieties with Medium versus High Hydration Capacity. Note: ${ }^{*}$ means $0.01 \leq P \leq 0.05,{ }^{* *}$ means $0.001 \leq P \leq 0.01,{ }^{* * *}$ means $P<0.001$. 
metabolites(ko01110), Ubiquinone and other terpenoid-quinone biosynthesis(ko00130), Phenylalanine metabolism (ko00360), Homologous recombination (ko03440), Pentose and glucuronate interconversions (ko00040), Glucosinolate biosynthesis (ko00966) and Benzoxazinoid biosynthesis (ko00402) (See Figure 3).

\subsection{PCA Analysis Result}

Principal component analysis (PCA) display the distance of the relationship between each sample, including visual effect of clusters groups. The PCA 3D Figure (See Figure 4) shows the genetic distance relationship between these genotypes generated by the DEGs. The closest genetic distance of these samples is between Farah and PBA Zahra and the second closest variety to Farah is AF06125. PBA Zahra and AF06125 are close in the same vertical line. The third closest variety to Farah is Nura, and then PBA Rana, and the most distant variety to Farah is PBA Warda. These results are perfectly matching the breeding selection pedigree information. Farah is PBA Zahra's paternal parent (50\%) and Farah is also in the pedigree of AF06125 (12.5\%). This figure also explains the much lower number of significant common pathways between PBA Zahra and AF06125; about one third of average pathways number compare to all other pairs comparison in Table 2.

\subsection{Validation Outcome}

Hydration Capacity The hydration capacity of AF06125 was highest with an average of $95.4 \%$ and PBA Zahra was lowest with $74.3 \%$. The data were for samples harvested from two sites growing in South Australia from 2007 to 2017. The other four samples were intermediate. (See Table 3) These results supported the pathway abundance of Hydration Capacity index samples information.

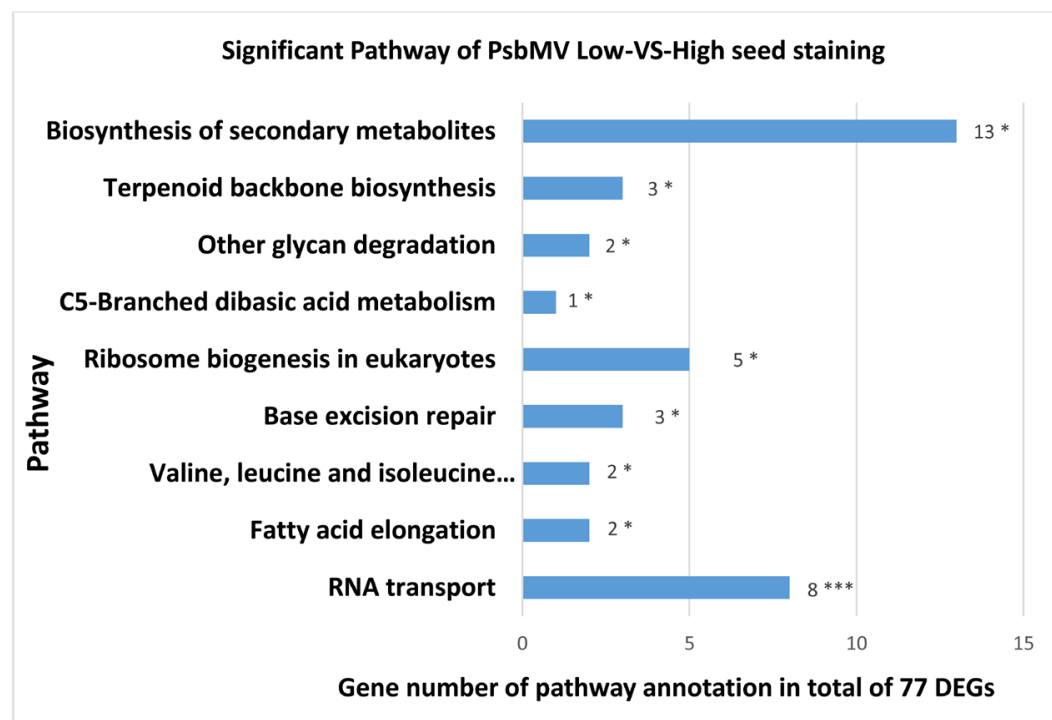

Figure 3. Significant pathways in faba bean seeds for the comparison of varieties with Low versus High seed staining due to infection of plants by PSbMV. Note: ${ }^{\star}$ means $0.01 \leq$ $P \leq 0.05,{ }^{* *}$ means $0.001 \leq P \leq 0.01,{ }^{* * *}$ means $P<0.001$. 


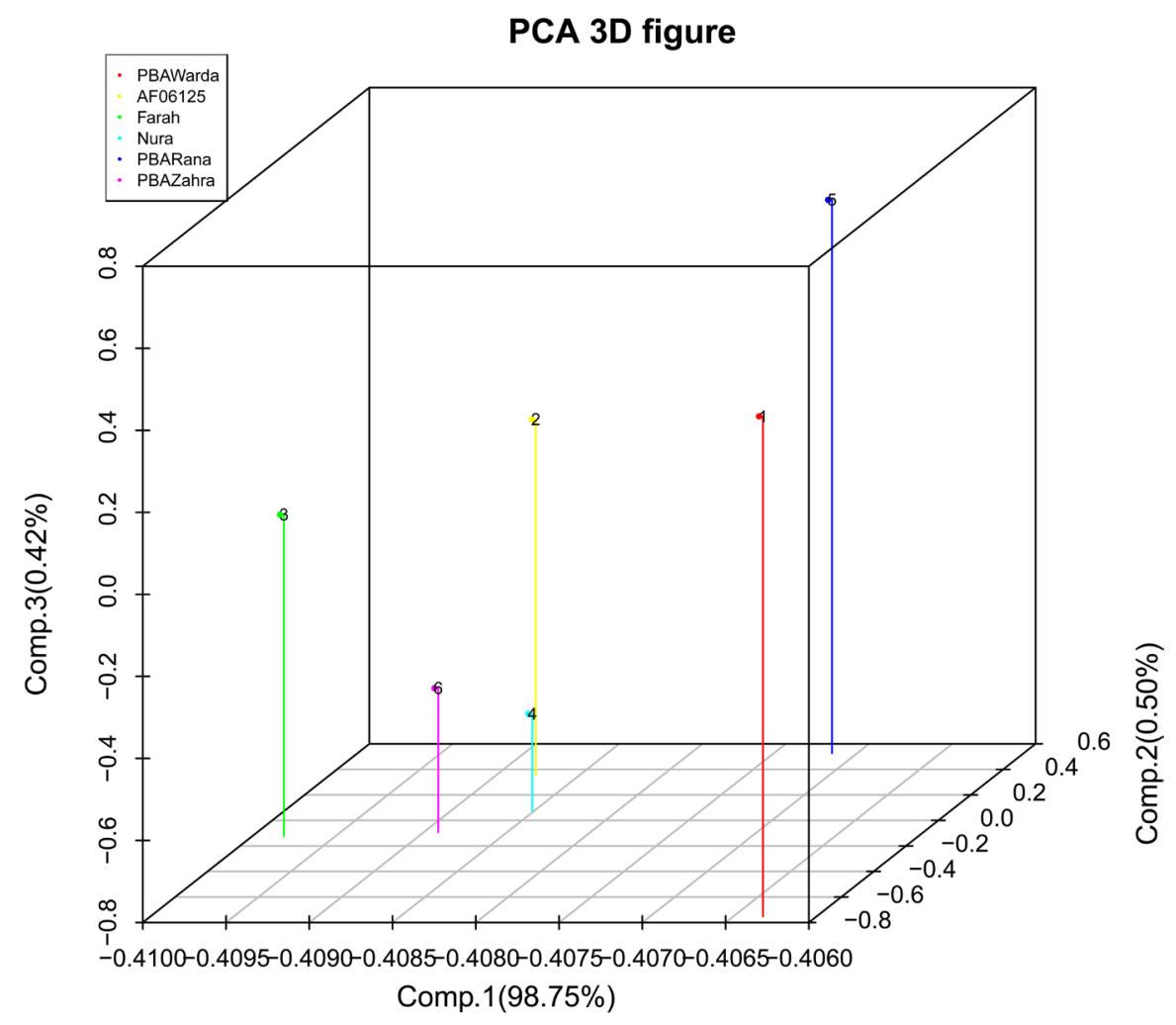

Figure 4. Three dimensional Principal Component (PCA) Analysis graph showing the relationship of genetic distance for six Australian faba bean varieties based on DEGs.

Table 3. Faba bean seeds Hydration Capacity from 2007 to 2017.

\begin{tabular}{ccccccc}
\hline Year & AF06125 & Farah & Nura & PBA Rana & PBA Warda & PBA Zahra \\
\hline 2017 & 94.37 & 81.53 & 82.59 & 88.79 & 85.17 & 71.5 \\
2016 & 93.56 & 81.6 & 79.42 & 75.88 & 84.58 & 72.11 \\
2015 & 100.5 & 85.1 & 93.6 & 99.8 & 97.8 & 81.1 \\
2014 & 90.6 & 77.7 & 81.5 & 79.9 & 86.3 & 73.8 \\
2013 & 90.9 & 73.4 & 78.8 & 71 & 77.7 & 55.6 \\
2012 & 96.2 & 79.6 & 87.6 & 88.4 & 90.1 & 71.6 \\
$2007-2011$ & 97.3 & 86.7 & 85.4 & 89 & 95 & 75.5 \\
Average & 94.8 & 80.8 & 84.1 & 84.7 & 88.1 & 71.6 \\
\hline
\end{tabular}

Data source: The data were tested samples which harvested from two sites: Freeling and Charlick in South Australia from 2007 to 2017.

PSbMV Index The PSbMV Index of AF06125 was lowest on 33\% and PBA Zahra was highest on $73 \%$, where the others were intermediate (See Table 4). The ELISA test and RT-PCR test were both positive for PSbMV virus indicating that all varieties are susceptible to PSbMV (See Table 5). These results confirmed the information of pathway abundance of PSbMV index samples information.

There are reports of pathway studies on faba bean at the seedling and flowering stages, however there are no prior reports of pathway studies on mature faba 
Table 4. Faba bean seeds PSbMV Index and its $t$ test result.

\begin{tabular}{ccccccc}
\hline t test & AF06125 & Warda & Farah & Nura & Rana & Zahra \\
\hline AF06125 & & & & & & \\
Warda & 0.0017 & & & & & \\
Farah & 0.0001 & 0.7786 & & & & \\
Nura & 0.0000 & 0.1048 & 0.1230 & & & \\
Rana & 0.0000 & 0.0285 & 0.0267 & 0.6566 & & \\
Zahra & 0.0000 & 0.0283 & 0.0302 & 0.3919 & 0.5622 & \\
PSbMV Index(Average) & 33 & 55 & 57 & 67 & 69 & 73 \\
St dev & 23.2 & 29.4 & 22.0 & 23.9 & 18.8 & 29.5 \\
\hline
\end{tabular}

Table 5. Result of testing virus inoculated and healthy controls of faba bean plants for PSbMV by PCR and ELISA.

\begin{tabular}{|c|c|c|c|c|}
\hline Sample ID & PSbMV Status & $\begin{array}{l}\text { PCR VT02/ } \\
03 \text { primers }\end{array}$ & $\begin{array}{c}\text { PSbMVQWF/ } \\
\text { R primers }\end{array}$ & $\begin{array}{c}\text { ELISA } \\
\text { result }\end{array}$ \\
\hline Nura-1 & inoculated & pos & pos & pos \\
\hline Nura-2 & inoculated & pos & pos & pos \\
\hline Nura-3 & inoculated & pos & pos & pos \\
\hline Nura-4 & inoculated & pos & pos & pos \\
\hline Nura-5 & inoculated & pos & pos & pos \\
\hline Nura-8, 9, 10, 11, 12 & pooled healthy control & neg & neg & neg \\
\hline Farah-1 & inoculated & pos & pos & pos \\
\hline Farah-2 & inoculated & neg & weak pos & neg \\
\hline Farah-3 & inoculated & pos & pos & pos \\
\hline Farah-4 & inoculated & pos & pos & pos \\
\hline Farah-5 & inoculated & pos & pos & pos \\
\hline Farah-8, 9, 10, 11, 12 & pooled healthy control & neg & neg & neg \\
\hline PBAZahra-1 & inoculated & pos & pos & pos \\
\hline PBAZahra-2 & inoculated & pos & pos & pos \\
\hline PBAZahra-3 & inoculated & pos & pos & pos \\
\hline PBAZahra-4 & inoculated & pos & pos & pos \\
\hline PBAZahra-5 & inoculated & pos & pos & pos \\
\hline PBAZahra-8, 9, 10, 11, 12 & pooled healthy control & neg & neg & neg \\
\hline PBARana-1 & inoculated & pos & pos & pos \\
\hline PBARana-2 & inoculated & pos & pos & pos \\
\hline PBARana-3 & inoculated & pos & pos & pos \\
\hline PBARana-4 & inoculated & pos & pos & pos \\
\hline PBARana-5 & inoculated & pos & pos & pos \\
\hline PBARana-8, 9, 10, 11, 12 & pooled healthy control & neg & neg & neg \\
\hline
\end{tabular}




\begin{tabular}{|c|c|c|c|c|}
\hline \multicolumn{5}{|l|}{ Continued } \\
\hline PBAWarda-1 & inoculated & pos & pos & pos \\
\hline PBAWarda-2 & inoculated & pos & pos & pos \\
\hline PBAWarda-3 & inoculated & pos & pos & pos \\
\hline PBAWarda-4 & inoculated & pos & pos & pos \\
\hline PBAWarda-5 & inoculated & pos & pos & pos \\
\hline PBAWarda- $8,9,10,11,12$ & pooled healthy control & neg & neg & neg \\
\hline AF06125-1 & inoculated & pos & pos & pos \\
\hline AF06125-2 & inoculated & pos & pos & pos \\
\hline AF06125-3 & inoculated & pos & pos & pos \\
\hline AF06125-4 & inoculated & pos & pos & pos \\
\hline AF06125-5 & inoculated & neg & weak pos & neg \\
\hline AF06125-8, 9, 10, 11, 12 & pooled healthy control & neg & neg & neg \\
\hline PSbMV-VT03F & \multicolumn{4}{|c|}{ GTG TTG GAG GAA TCA CAC CAG AAG AAT GTG } \\
\hline PSbMV-VT02R & \multicolumn{4}{|c|}{ GCA GTT GCT ACA TCC ATC ATT GTT GGC CAT) } \\
\hline \multicolumn{5}{|l|}{ Amplicon size $1100 \mathrm{bp}$} \\
\hline PSbMVQW-F & \multicolumn{4}{|c|}{ GTGTTGGAGGAATCACACGA } \\
\hline PSbMVQW-R & \multicolumn{4}{|c|}{ GGTGTTGTTTCCCATGTCTGT } \\
\hline
\end{tabular}

bean seeds. Hence the pathways information reported here is the first deep pathway analysis of faba bean seeds RNA-Seq. It can be a source of knowledge for future studies on faba bean seeds. These results reveal the genetic distance within these varieties and genomic information for faba bean seeds, and fill the knowledge gaps of faba bean seed pathways.

The KEGG pathways information will help understanding the faba bean seed genes functional activities. The genetic distance of these varieties are confirmed both by DEGs generated from RNA-Seq and breeding pedigree information. There are pathway analysis studies on other plants and crops such as rice, maize, and chickpeas.

Due to the limited number of faba bean genotypes using for sequencing in this report, it may not fully identify all the pathways information in the faba bean seeds. Hence, investigating more genotypes in future sequencing for analysis would be recommended.

RNA-Seq (Quantification) is a cost-effective quantification method that produces high reproducibility, high accuracy and wide dynamic range. It can be applied in drug response, biomarker detection, basic medical research, and drug $\mathrm{R} \& \mathrm{D}$. And also applied in gene expression analysis, differential gene expression analysis, expression profile analysis of DEGs, and Gene ontology classification and pathway enrichment analysis. 


\section{Conclusion}

In summary, there are a total of 75 significant or highly significant KEGG pathways discovered within these faba beans seeds RNA-Seq. There are a total of 9 significant pathways (over 53.3\% pairs) that are conserved in abundance within all the seeds, 41 significant pathways found within 2 to 6 pair comparisons and 25 significant pathways were unique to single pair comparisons. There were 8 specific significant pathways associated with the faba bean seed Hydration Capacity trait and 9 different specific significant pathways associated with the PSbMV seeds staining trait. The seeds hydration level has been validated by 10 years of standard hydration capacity testing. The seeds staining level of these samples has been validated by the field observation in South Australia and a growth room inoculation test in Adelaide. ELISA and RT-PCR experiment confirmed that inoculated plants were positive for PSbMV. The genetic distance between these varieties in the PCA 3D graph confirmed breeding pedigree selection information.

\section{Ethics Approval and Consent to Participate}

Not applicable.

\section{Consent for Publication}

Not applicable.

\section{Availability of Data and Materials}

BioProject ID PRJNA319071 and RNA-Seq (Quantification) data are deposited at National Centre for Biotechnology Information (NCBI) gene bank. Reference number is SRA accession: SRP074308. The faba bean Assembly data deposited in Figshare: The data DOI is: Digital Object Identifier 10.6084/m9.figshare.4910039.

\section{Authors' Contributions}

S.Y. Yang designed the experiment and wrote the manuscript, Y. Wang and J. Li carried for all sequences data analysis. N. Habili and Q. Wu done the PSbMV RT-PCR validation test. J.G. Paull commented and revised the manuscript.

\section{Acknowledgements}

We would like to thanks Dr. Joop Van Leur and Ms. Jule George, NSW Department of Primary Industries for the PSbMV validation of ELISA test. We also would like to thank Dr. Bujun Shi for suggestion on the validation test and comments on the draft. We would like to thanks Angela Mills, Librarian of University of Adelaide, who helps to fix the reference correctly.

\section{Funding}

We acknowledge support from University of Adelaide and Grains Research \& 
Development Corporation (GRDC) for the study.

\section{Conflicts of Interest}

The authors declare no conflicts of interest regarding the publication of this paper.

\section{References}

[1] Zohary, D. and Hopf, M. (1973) Domestication of Pulses in the Old World: Legumes Were Companions of Wheat and Barley When Agriculture Began in the Near East. Science, 182, 887-894. https://doi.org/10.1126/science.182.4115.887

[2] FAOSTAT. http://faostat.fao.org

[3] Johnston, J.S., Bennett, M.D., Rayburn, A.L., Galbraith, D.W. and Price, H.J. (1999) Reference Standards for Determination of DNA Content of Plant Nuclei. American Journal of Botany, 86, 609-613. https://doi.org/10.2307/2656569

[4] Xu, H., Gao, Y. and Wang, J. (2012) Transcriptomic Analysis of Rice (Oryza sativa) Developing Embryos Using the RNA-Seq Technique. PLoS ONE, 7, e30646.

https://doi.org/10.1371/journal.pone.0030646

[5] Davidson, R.M., Hansey, C.N., Gowda, M., Childs, K.L., Lin, H., Vaillancourt, B., Sekhon, R.S., de Leon, N., Kaeppler, S.M., Jiang, N. and Buell, C.R. (2011) Utility of RNA Sequencing for Analysis of Maize Reproductive Transcriptomes. The Plant Genome, 4, 191-203. https://doi.org/10.3835/plantgenome2011.05.0015

[6] Kakumanu, A., Ambavaram, M.M.R., Klumas, C., Krishnan, A., Batlang, U., Myers, E., Grene, R. and Pereira, A. (2012) Effects of Drought on Gene Expression in Maize Reproductive and Leaf Meristem Tissue Revealed by RNA-Seq. Plant Physiology, 160, 846-867. https://doi.org/10.1104/pp.112.200444

[7] Garg, R., Patel, R.K., Tyagi, A.K. and Jain, M. (2011) De Novo Assembly of Chickpea Transcriptome Using Short Reads for Gene Discovery and Marker Identification. DNA Research, 18, 53-63. https://doi.org/10.1093/dnares/dsq028

[8] Hiremath, P.J., Farmer, A., Cannon, S.B., Woodward, J., Kudapa, H., Tuteja, R., Kumar, A., BhanuPrakash, A., Mulaosmanovic, B., Gujaria, N., Krishnamurthy, L., Gaur, P.M., KaviKishor, P.B., Shah, T., Srinivasan, R., Lohse, M., Xiao, Y., Town, C.D., Cook, D.R., May, G.D. and Varshney, R.K. (2011) Large-Scale Transcriptome Analysis in Chickpea (Cicer arietinum L.), an Orphan Legume Crop of the Semi-Arid Tropics of Asia and Africa. Plant Biotechnology Journal, 9, 922-931. https://doi.org/10.1111/j.1467-7652.2011.00625.x

[9] Sudheesh, S., Sawbridge, T.I., Cogan, N.O., Kennedy, P., Forster, J.W. and Kaur, S. (2015) De Novo Assembly and Characterisation of the Field Pea Transcriptome Using RNA-Seq. BMC Genomics, 16, 611. https://doi.org/10.1186/s12864-015-1815-7

[10] Kaur, S., Pembleton, L.W., Cogan, N.O., Savin, K.W., Leonforte, T., Paull, J., Materne, M. and Forster, J.W. (2012) Transcriptome Sequencing of Field Pea and Faba Bean for Discovery and Validation of SSR Genetic Markers. BMC Genomics, 13, 104. https://doi.org/10.1186/1471-2164-13-104

[11] Wu, G., Zhang, L., Yin, Y., Wu, J., Yu, L., Zhou, Y. and Li, M. (2015) Sequencing, De Novo Assembly and Comparative Analysis of Raphanus sativus Transcriptome. Frontiers in Plant Science, 6, 198. https://doi.org/10.3389/fpls.2015.00198

[12] Arun Chinnappa, K.S. and McCurdy, D.W. (2015) De Novo Assembly of a Cotyledon-Enriched Transcriptome Map of Vicia faba (L.) for Transfer Cell Research. Frontiers in Plant Science, 6. https://doi.org/10.3389/fpls.2015.00217 
[13] Khan, M.A., Alghamdi, S.S., Ammar, M.H., Sun, Q., Teng, F., Migdadi, H.M. and Al-Faifi, S.A. (2019) Transcriptome Profiling of Faba Bean (Vicia faba L.) Drought-Tolerant Variety Hassawi-2 under Drought Stress Using RNA Sequencing. Electronic Journal of Biotechnology, 39, 15-29. https://doi.org/10.1016/j.ejbt.2019.02.004

[14] Cooper, J.W., Wilson, M.H., Derks, M.F.L., Smit, S., Kunert, K.L., Cullis, C. and Foyer, C.H. (2017) Enhancing Faba Bean (Vicia faba L.) Genome Resources. Journal of Experimental Botany, 68, 1941-1953. https://doi.org/10.1093/jxb/erx117

[15] O’Sullivan, D.M. and Angra, D. (2016) Advances in Faba Bean Genetics and Genomics. Frontiers in Genetics, 7, 150. https://doi.org/10.3389/fgene.2016.00150

[16] Yang, S.Y., Wilkinson, M.J., Wang, Y., Li, J. and Paull, J.G. (2017) Gene Function Expression Profile of Faba Bean (Vicia faba) Seeds. Journal of Applied Microbiology and Biochemistry, 1, 3-11.

[17] Pazhamala, L.T., Purohit, S., Saxena, R.K., Garg, V., Krishnamurthy, L., Verdier, J., and Varshney, R.K. (2017) Gene Expression Atlas of Pigeonpea and Its Application to Gain Insights into Genes Associated with Pollen Fertility Implicated in Seed Formation. Journal of Experimental Botany, 68, 2037-2054.

https://doi.org/10.1093/jxb/erx010

[18] Chien, C.H., Chow, C.N., Wu, N.Y., Chiang-Hsieh, Y.F., Hou, P.F. and Chang, W.C. (2015) EXPath: A Database of Comparative Expression Analysis Inferring Metabolic Pathways for Plants. BMC Genomics, 16, S6. https://doi.org/10.1186/1471-2164-16-S2-S6

[19] Lai, L.M., Liberzon, A., Hennessey, J., Jiang, G.X., Qi, J.L., Mesirov, J.P. and Ge, S.X. (2012) AraPath: A Knowledgebase for Pathway Analysis in Arabidopsis. Bioinfor matics, 28, 2291-2292. https://doi.org/10.1093/bioinformatics/bts421

[20] Masclaux, F.G., Bruessow, F., Schweizer, F., Gouhier-Darimont, C., Keller, L. and Reymond, P. (2012) Transcriptome Analysis of Intraspecific Competition in Arabidopsis thaliana Reveals Organ-Specific Signatures Related to Nutrient Acquisition and General Stress Response Pathways. BMC Plant Biology, 12, 227. https://doi.org/10.1186/1471-2229-12-227

[21] Ma, S.S., Shah, S., Bohnert, H.J., Snyder, M. and Dinesh-Kumar, S.P. (2013) Incorporating Motif Analysis into Gene Co-Expression Networks Reveals Novel Modular Expression Pattern and New Signaling Pathways. PLoS Genetics, 9, e1003840. https://doi.org/10.1371/journal.pgen.1003840

[22] Kohan-Baghkheirati, E. and Geisler-Lee, J. (2015) Gene Expression, Protein Function and Pathways of Arabidopsis thaliana Responding to Silver Nanoparticles in Comparison to Silver Ions, Cold, Salt, Drought, and Heat. Nanomaterials, 5, 436-467. https://doi.org/10.3390/nano5020436

[23] Lang, C. and Xi, J. (2016) Bioinformatics Identification of the Methylerythritol Phosphate Pathway Associated Genes in Arabidopsis thaliana with ceh1 Mutant. Russian Journal of Plant Physiology, 63, 293-299. https://doi.org/10.1134/S1021443716020096

[24] Oono, Y., Yazawa, T., Kawahara, Y., Kanamori, H., Kobayashi, F., Sasaki, H., Mori, S., Wu, J.Z., Handa, H., Itoh, T. and Matsumoto, T. (2014) Genome-Wide Transcriptome Analysis Reveals That Cadmium Stress Signaling Controls the Expression of Genes in Drought Stress Signal Pathways in Rice. PLoS ONE, 9, e96946. https://doi.org/10.1371/journal.pone.0096946

[25] Benevenuto, R.F., Agapito-Tenfen, S.Z., Vilperte, V., Wikmark, O.G., van Rensburg, P.J. and Nodari, R.O. (2017) Molecular Responses of Genetically Modified Maize to 
Abiotic Stresses as Determined through Proteomic and Metabolomic Analyses. PLoS ONE, 12, e0173069. https://doi.org/10.1371/journal.pone.0173069

[26] Chen, T.T., Zhang, L., Shang, H.H., Liu, S.D., Peng, J., Gong, W.K., Shi, Y.Z., Zhang, S.P., Li, J.W., Gong, J.W., Ge, Q., Liu, A.Y., Ma, H.J., Zhao, X.H. and Yuan, Y.L. (2016) iTRAQ-Based Quantitative Proteomic Analysis of Cotton Roots and Leaves Reveals Pathways Associated with Salt Stress. PLoS ONE, 11, e0148487. https://doi.org/10.1371/journal.pone.0148487

[27] Cheng, Y.Q., Zhao, Y.X., Liu, J.F., Yang, B.W. and Ming, Y.T. (2017) Comparison of Phytohormone Biosynthesis and Signal Transduction Pathways in Developing and Abortive Hazelnut Ovules. Plant Growth Regulation, 81, 147-157. https://doi.org/10.1007/s10725-016-0196-5

[28] Wang, R., Xu, S., Wang, N., Xia, B., Jiang, Y.M. and Wang, R. (2017) Transcriptome Analysis of Secondary Metabolism Pathway, Transcription Factors, and Transporters in Response to Methyl Jasmonate in Lycoris aurea. Frontiers in Plant Science, 7, 1971. https://doi.org/10.3389/fpls.2016.01971

[29] Kaur, S., Cogan, N., Forster, J. and Paull, J. (2014) Assessment of Genetic Diversity in Faba Bean Based on Single Nucleotide Polymorphism. Diversity, 6, 88-101. https://doi.org/10.3390/d6010088

[30] Mortazavi, A., Williams, B.A., McCue, K., Schaeffer, L. and Wold, B. (2008) Mapping and Quantifying Mammalian Transcriptomes by RNA-Seq. Nature Methods, 5, 621-628. https://doi.org/10.1038/nmeth.1226

[31] Wang, Z., Gerstein, M. and Snyder, M. (2009) RNA-Seq: A Revolutionary Tool for Transcriptomics. Nature Reviews Genetics, 10, 57-63. https://doi.org/10.1038/nrg2484

[32] Ozsolak, F. and Milos, P.M. (2010) RNA Sequencing: Advances, Challenges and Opportunities. Nature Reviews Genetics, 12, 87. https://doi.org/10.1038/nrg2934

[33] Patro, R., Mount, S.M. and Kingsford, C. (2014) Sailfish Enables Alignment-Free Isoform Quantification from RNA-seq Reads Using Lightweight Algorithms. Nature Biotechnology, 32, 462-464. https://doi.org/10.1038/nbt.2862

[34] de Hoon, M.J., Imoto, S., Nolan, J. and Miyano, S. (2004) Open Source Clustering Software. Bioinformatics, 20, 1453-1454. https://doi.org/10.1093/bioinformatics/bth078

[35] Eisen, M.B., Spellman, P.T., Brown, P.O. and Botstein, D. (1998) Cluster Analysis and Display of Genome-Wide Expression Patterns. Proceedings of the National Academy of Sciences of the United States of America, 95, 14863-14868. https://doi.org/10.1073/pnas.95.25.14863

[36] Saldanha, A.J. (2004) Java Treeview-Extensible Visualization of Microarray Data. Bioinformatics, 20, 3246-3248. https://doi.org/10.1093/bioinformatics/bth349

[37] Audic, S. and Claverie, J.-M. (1997) The Significance of Digital Gene Expression Profiles. Genome Research, 7, 986-995. https://doi.org/10.1101/gr.7.10.986

[38] Abdi, H. (2007) The Bonferonni and Šidák Corrections for Multiple Comparisons. In: Salkind, N.J., Ed., Encyclopedia of Measurement and Statistics, 3rd Edition, Sage, Thousand Oaks, 103-107.

[39] Benjamini, Y. and Yekutieli, D. (2001) The Control of the False Discovery Rate in Multiple Testing under Dependency. Annals of Statistics, 29, 1165-1188. https://doi.org/10.1214/aos/1013699998

[40] Aakanksha, W., Nigam, V.K. and Pandey, D.M. (2014) Understanding New Paradigm of Plant Metabolomes through Secondary Metabolite Enrichment. Research 
Journal of Biotechnology, 9, 90-98.

[41] Tarazona, S., Garcia-Alcalde, F., Dopazo, J., Ferrer, A. and Conesa, A. (2011) Differential Expression in RNA-seq: A Matter of Depth. Genome Research, 21, 2213-2223. https://doi.org/10.1101/gr.124321.111

\section{List of Abbreviations}

RNA-Seq: RNA sequencing

PSbMV: Pea seed-borne mosaic virus

DEGs: Differentially Expressed Genes

KEGG: Kyoto Encyclopedia of Genes and Genomes

ELISA: Enzyme-linked immunosorbent assay

PCA: Principal Component Analysis 


\section{Supplementary Files}

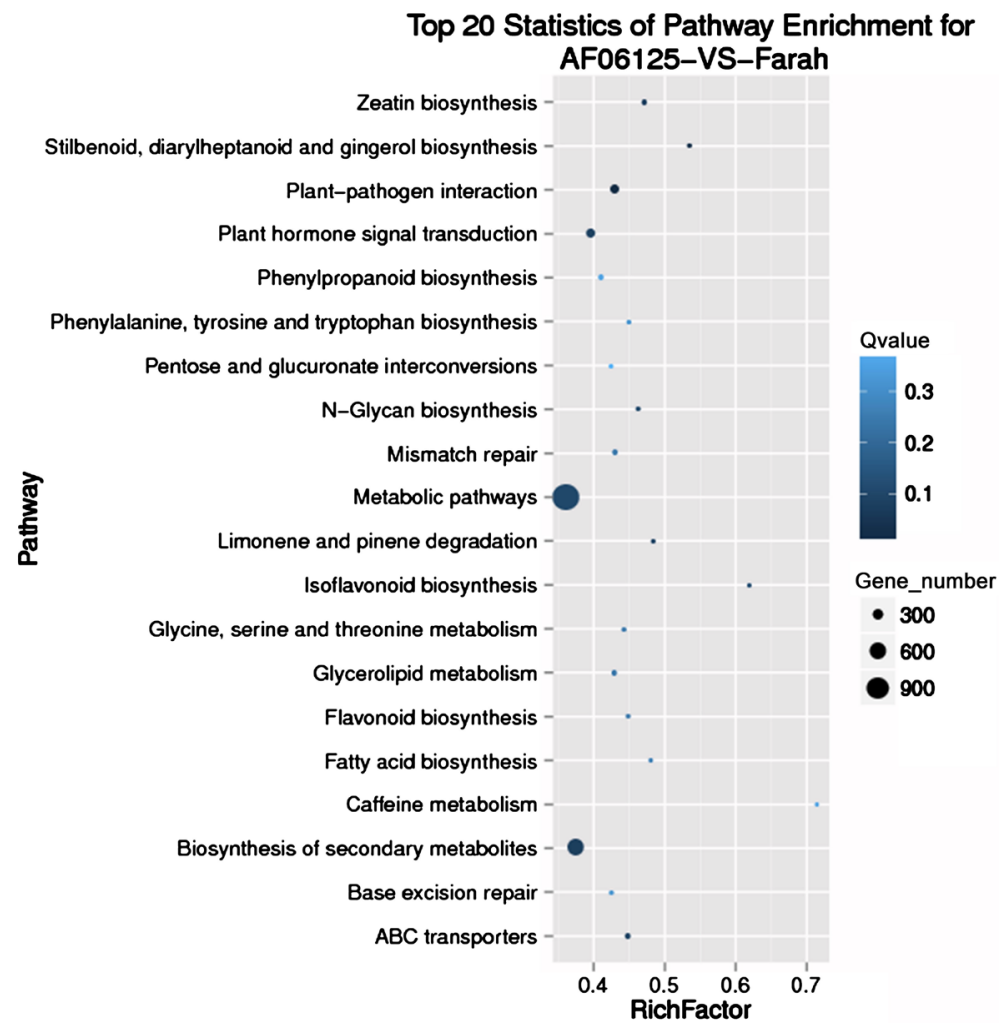

Figure S1. AF06125-VS-Farah.path.enrichment.

Top 20 Statistics of Pathway Enrichment for AF06125-VS-Nura

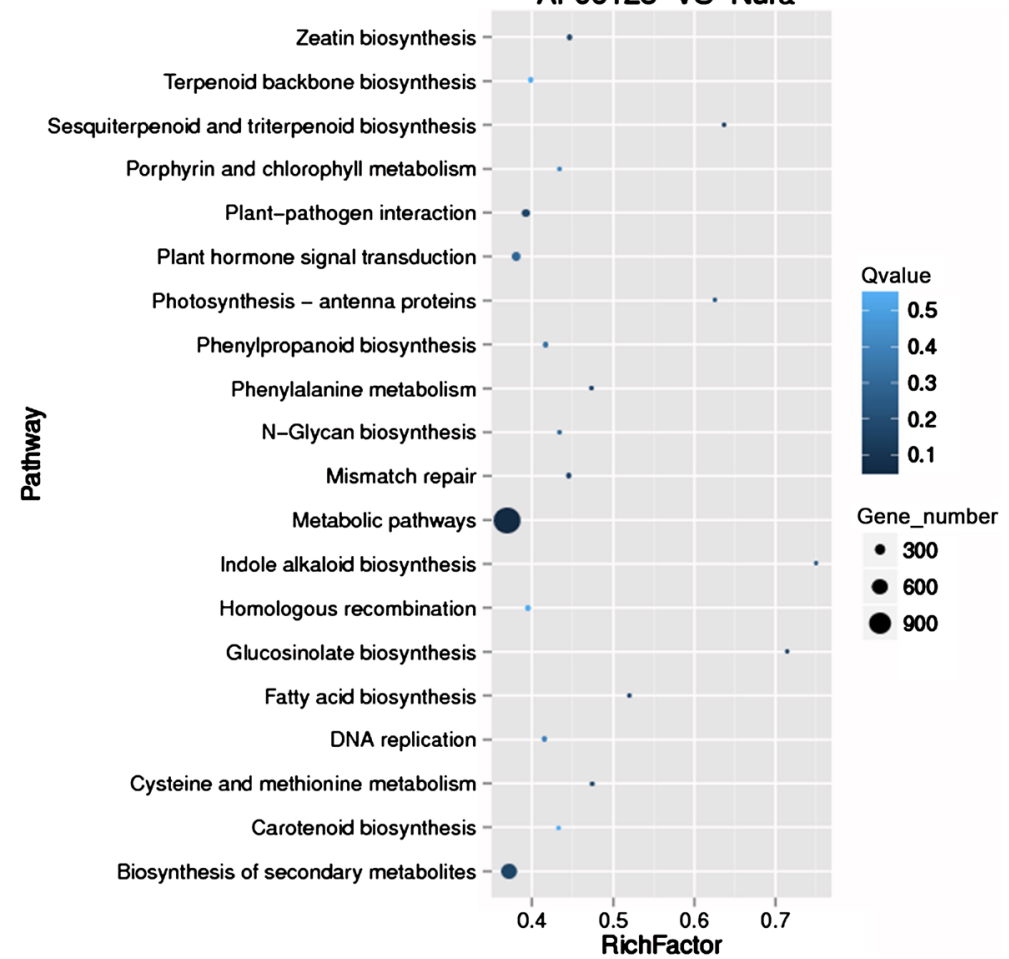

Figure S2. AF06125-VS-Nura.path.enrichment. 


\section{Top 20 Statistics of Pathway Enrichment for} AF06125-VS-PBARana

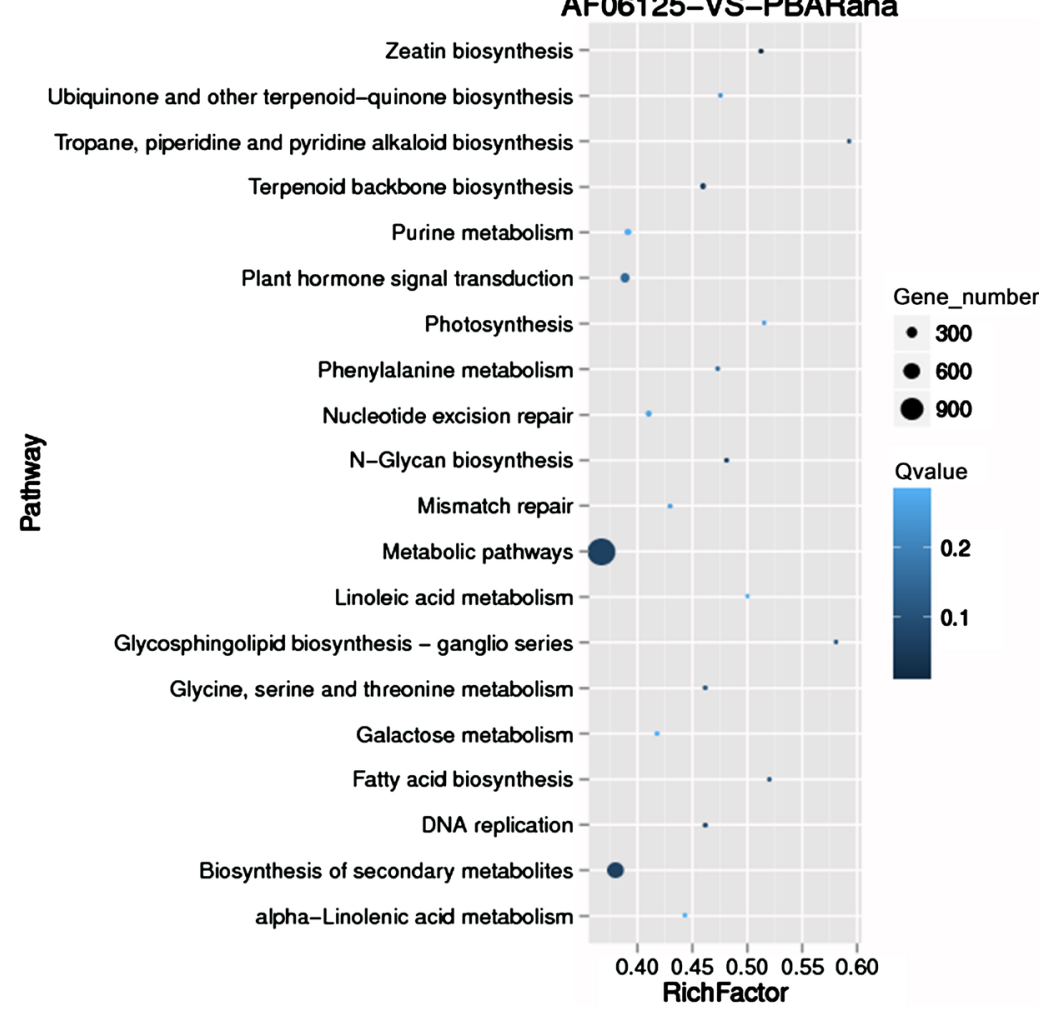

Figure S3. AF06125-VS-PBARana.path.enrichment.

Top 20 Statistics of Pathway Enrichment for AF06125-VS-PBAWarda

Zeatin biosynthesis

Terpenoid backbone biosynthesis

Starch and sucrose metabolism

Sphingolipid metabolism -

Selenocompound metabolism -

Plant hormone signal transduction -

Phenylpropanoid biosynthesis

Phenylalanine metabolism -

Pentose phosphate pathway

Metabolic pathways

离

Limonene and pinene degradation

Glycosphingolipid biosynthesis - ganglio series

Glycine, serine and threonine metabolism

Glutathione metabolism -

Fatty acid elongation -

Endocytosis

Cyanoamino acid metabolism -

Biosynthesis of secondary metabolites -

Benzoxazinoid biosynthesis

Amino sugar and nucleotide sugar metabolism

$0.4 \quad 0.5$

Gene_number

- 250

- 500

750

1000

Qvalue

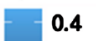

0.3

0.2

0.1

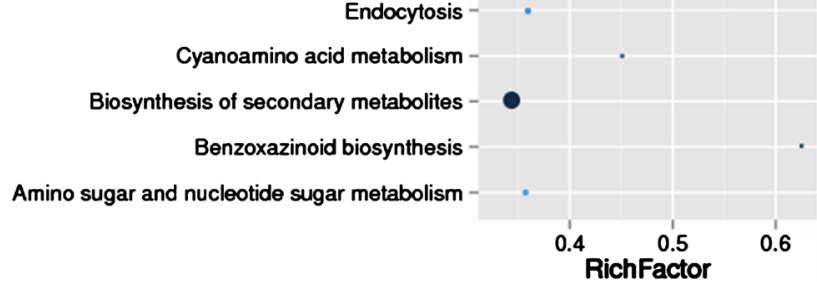

Figure S4. AF06125-VS-PBAWarda.path.enrichment. 
Top 20 Statistics of Pathway Enrichment for Farah-VS-PBARana

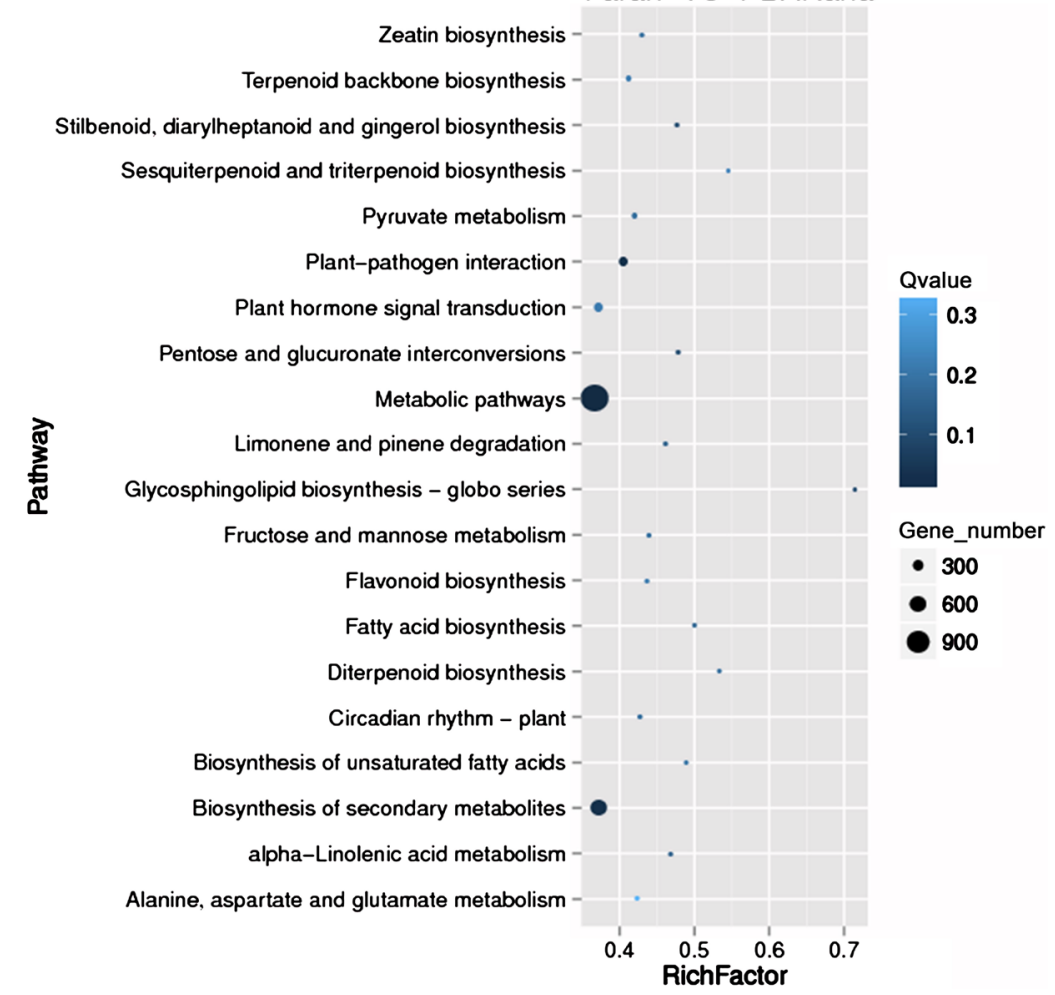

Figure S5. Farah-VS-PBARana.path.enrichment.

\section{Top 20 Statistics of Pathway Enrichment for Farah-VS-PBAWarda}

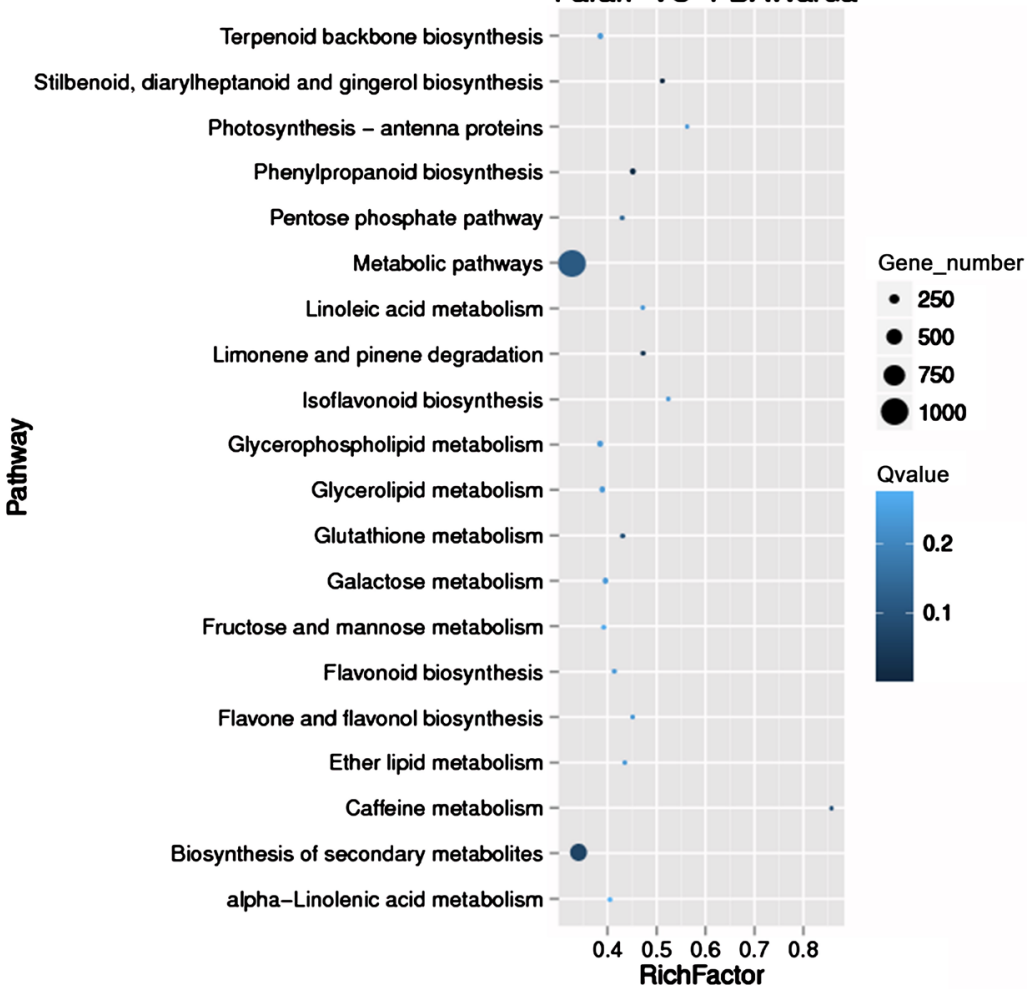

Figure S6. Farah-VS-PBAWarda.path.enrichment. 


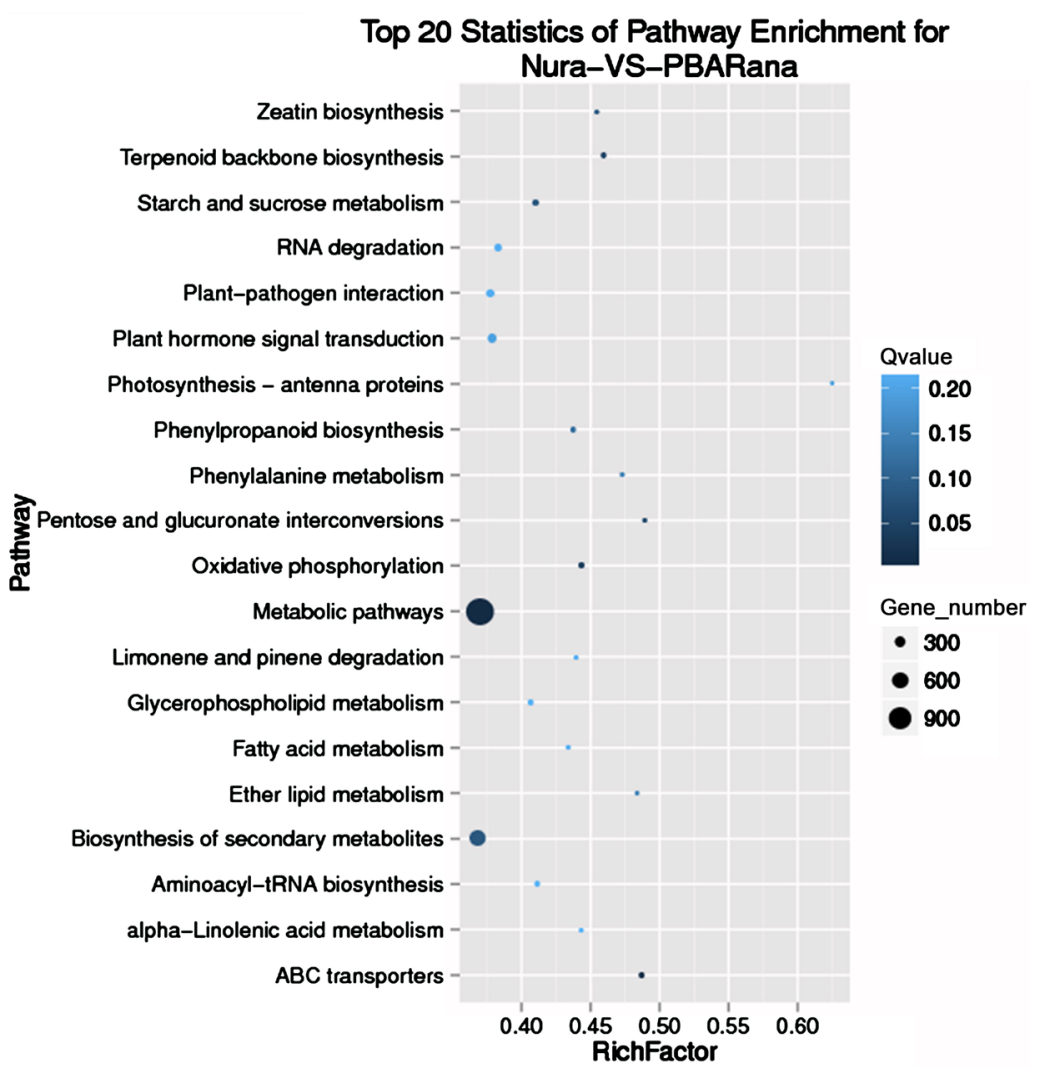

Figure S7. Nura-VS-PBARana.path.enrichment.

Top 20 Statistics of Pathway Enrichment for Nura-VS-PBAWarda

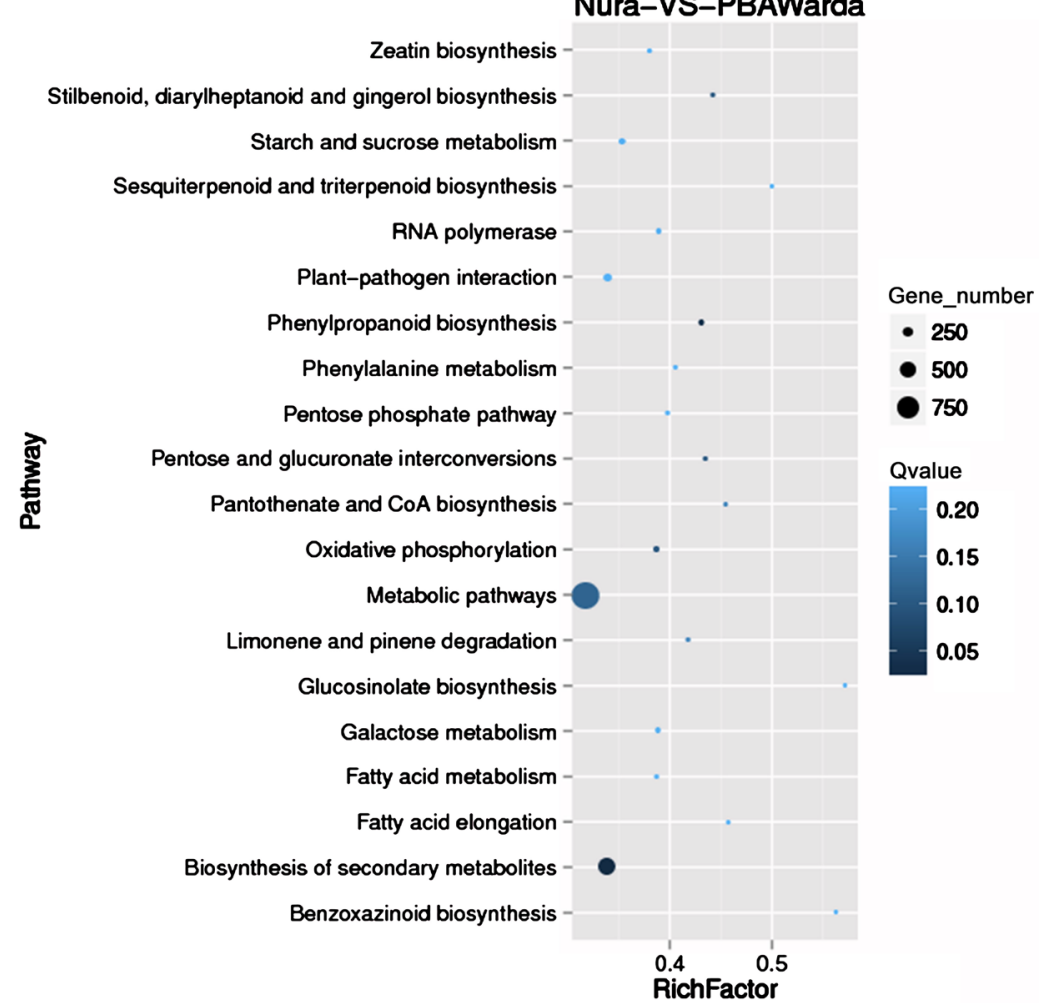

Figure S8. Nura-VS-PBAWarda.path.enrichment. 
Top 20 Statistics of Pathway Enrichment for PBARana-VS-PBAWarda

Zeatin biosynthesis -

Ubiquinone and other terpenoid-quinone biosynthesis -

Terpenoid backbone biosynthesis

Stilbenoid, diarylheptanoid and gingerol biosynthesis -

Riboflavin metabolism -

Phenylpropanoid biosynthesis

Peroxisome

Pentose and glucuronate interconversions -

Metabolic pathways -

离

Linoleic acid metabolism -

Limonene and pinene degradation -

Glycosphingolipid biosynthesis - ganglio series -

Fructose and mannose metabolism

Flavone and flavonol biosynthesis -

Diterpenoid biosynthesis

Cysteine and methionine metabolism

Caffieine metabolism

Butanoate metabolism

Biosynthesis of secondary metabolites -

ABC transporters

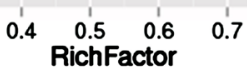

Gene_number

- 250

- 500

750

1000

Qvalue
-0.4

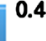

0.3

0.2

0.1

Figure S9. PBARana-VS-PBAWarda.path.enrichment.

Top 20 Statistics of Pathway Enrichment for PBAZahra-VS-AF06125

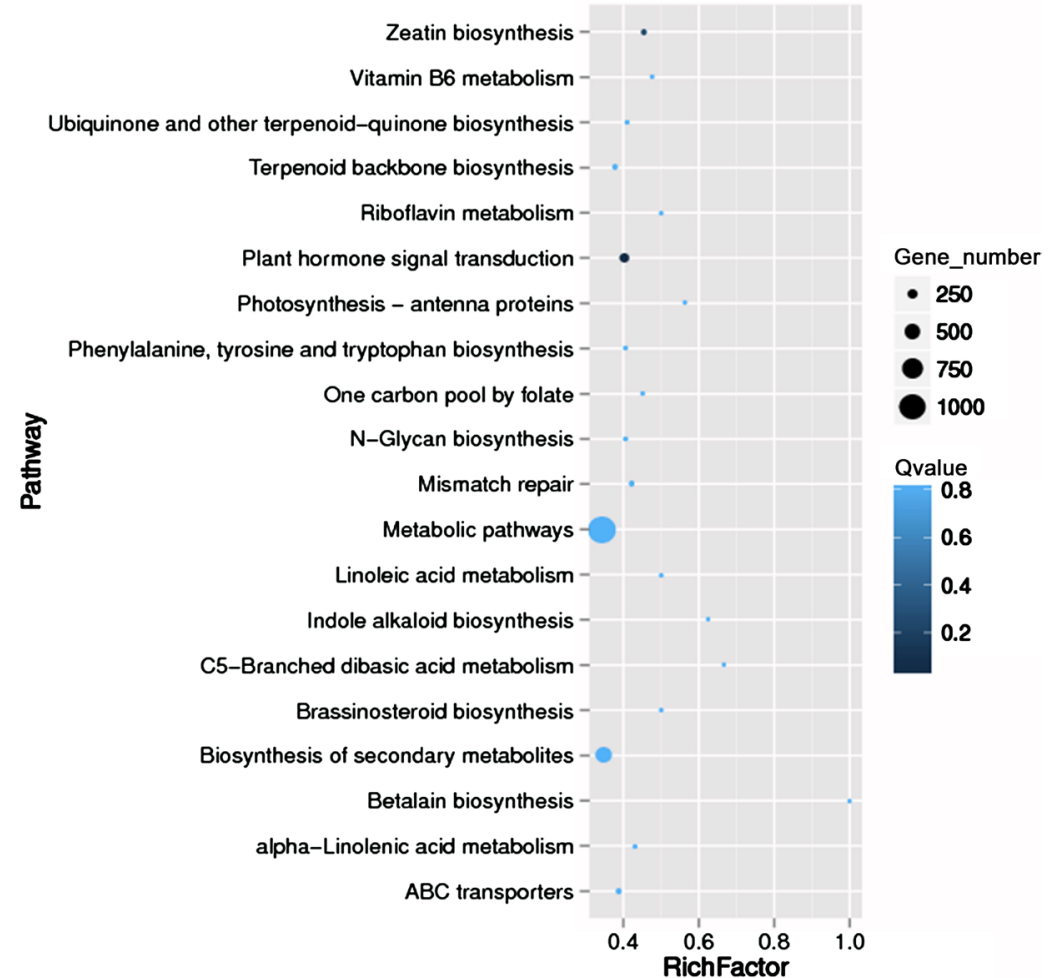

Figure S10. PBAZahra-VS-AF06125.path.enrichment. 


\section{Top 20 Statistics of Pathway Enrichment for PBAZahra-VS-Farah}

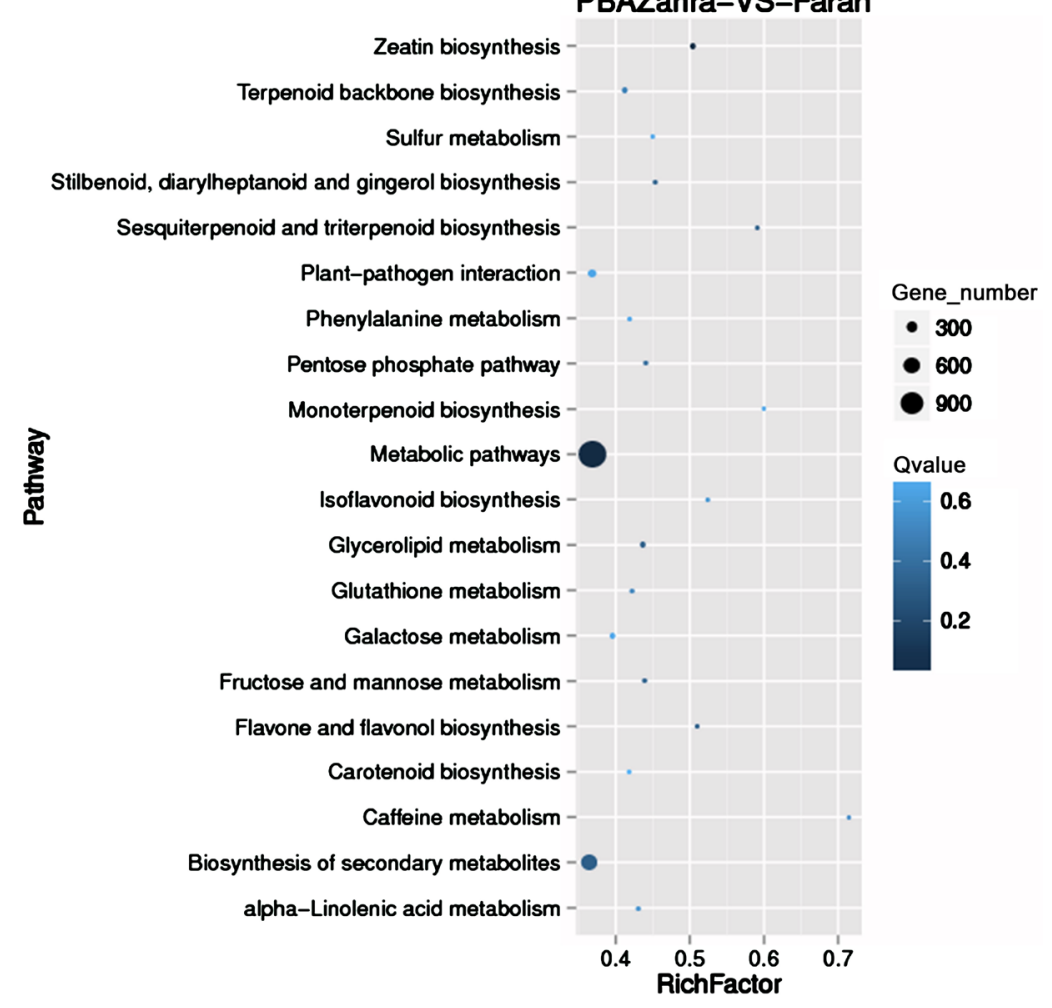

Figure S11. PBAZahra-VS-Farah.path.enrichment.

Top 20 Statistics of Pathway Enrichment for PBAZahra-VS-Nura

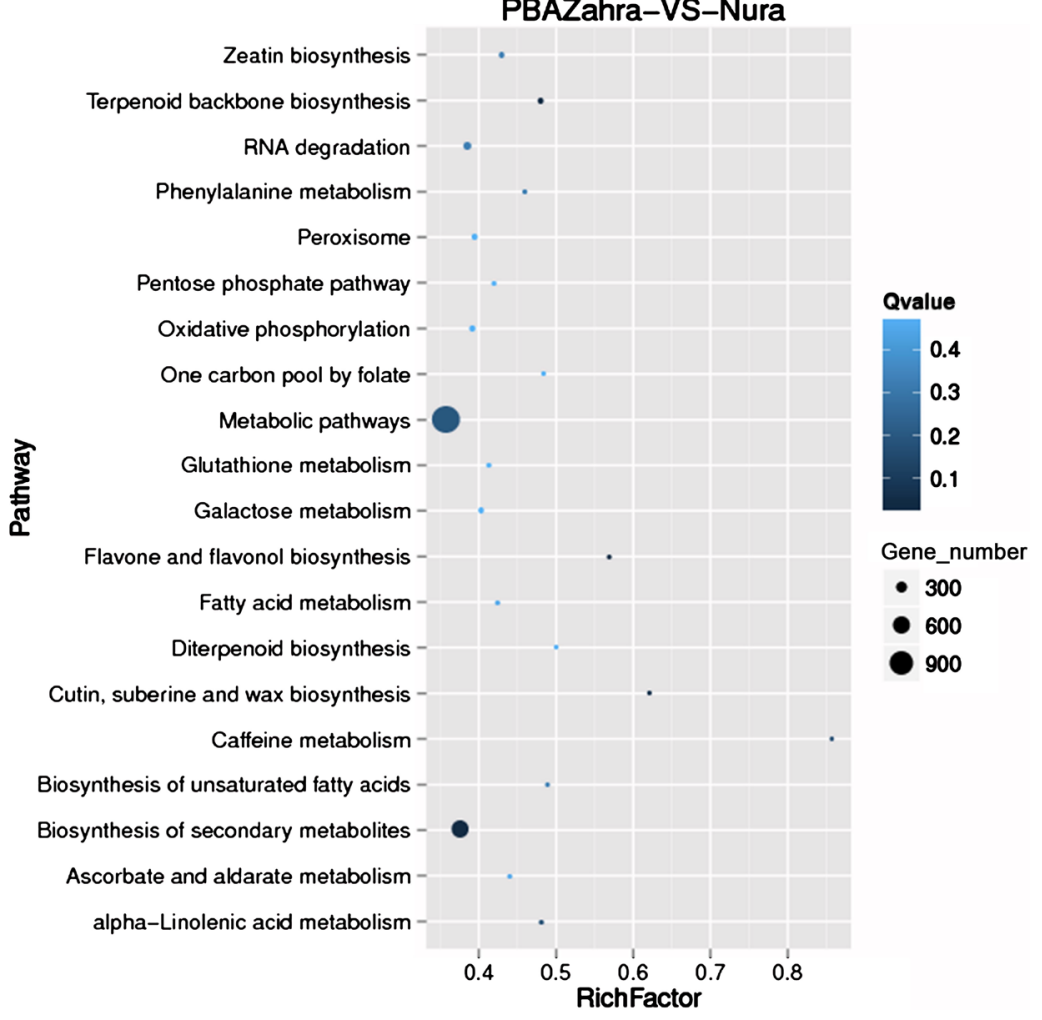

Figure S12. PBAZahra-VS-Nura.path.enrichment. 
Top 20 Statistics of Pathway Enrichment for PBAZahra-VS-PBARana

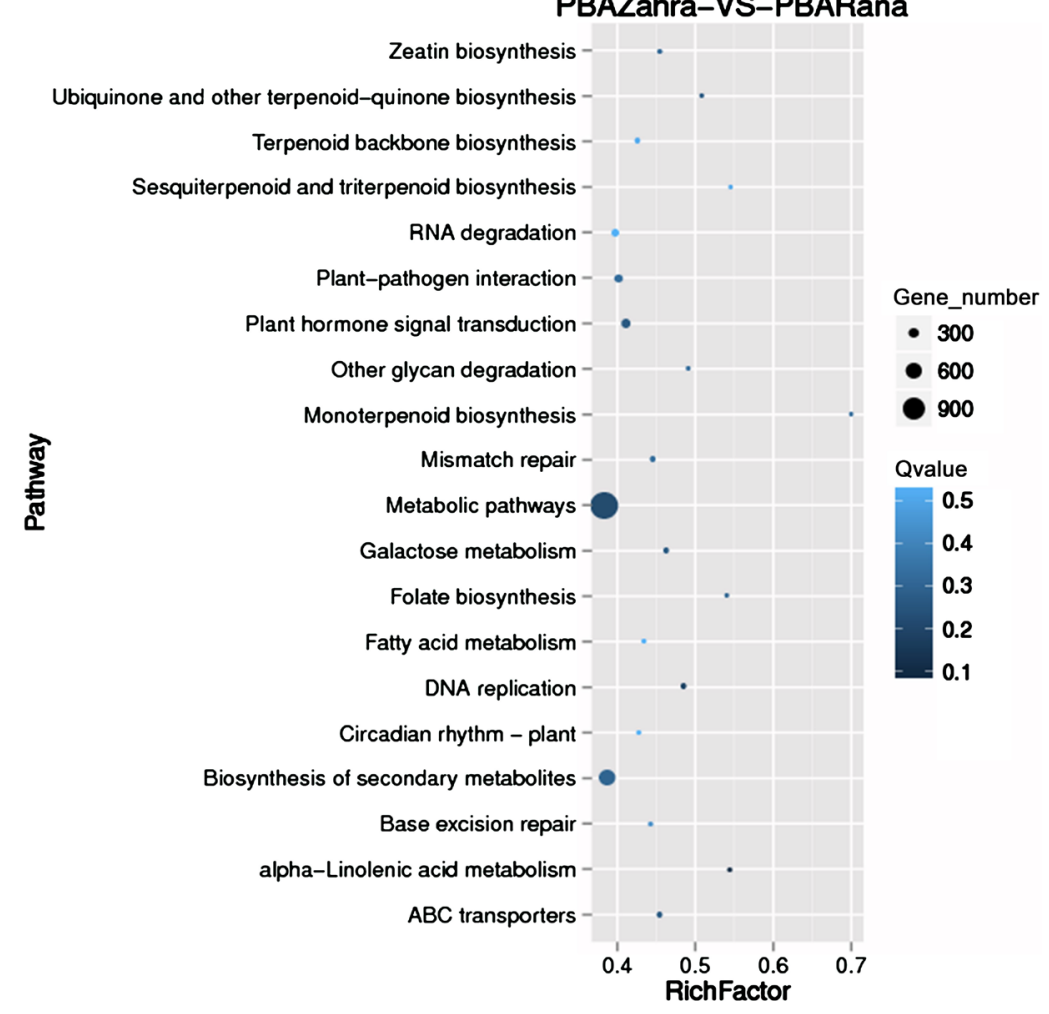

Figure S13. PBAZahra-VS-PBARana.path.enrichment.

Top 20 Statistics of Pathway Enrichment for PBAZahra-VS-PBAWarda

Zeatin biosynthesis -

Stilbenoid, diarylheptanoid and gingerol biosynthesis

Sphingolipid metabolism

Plant hormone signal transduction -

Photosynthesis

Phenylpropanoid biosynthesis

Pentose and glucuronate interconversions

Metabolic pathways

Linoleic acid metabolism

帘

Limonene and pinene degradation

Glycosphingolipid biosynthesis - ganglio series

Galactose metabolism

Flavonoid biosynthesis

Flavone and flavonol biosynthesis

Fatty acid elongation

Endocytosis

Cyanoamino acid metabolism

Biosynthesis of unsaturated fatty acids

Biosynthesis of secondary metabolites

alpha-Linolenic acid metabolism

Gene_number

- 250

- 500

750

1000

Qvalue

\section{$\begin{array}{llll}0.4 & 0.5 & 0.6 & 0.7\end{array}$ \\ RichFactor}

Figure S14. PBAZahra-VS-PBAWarda.path.enrichment. 